\title{
REFLECTING BROWNIAN MOTION IN A CUSP
}

\author{
R. DANTE DEBLASSIE AND ELLEN H. TOBY
}

\begin{abstract}
Let $C$ be the cusp $\left\{(x, y): x \geq 0,-x^{\beta} \leq y \leq x^{\beta}\right\}$ where $\beta>1$. Set $\partial C_{1}=\left\{(x, y): x \geq 0, y=-x^{\beta}\right\}$ and $\partial C_{2}=\{(x, y): x \geq$ $\left.0, y=x^{\beta}\right\}$. We study the existence and uniqueness in law of reflecting Brownian motion in $C$. The angle of reflection at $\partial C_{j} \backslash\{0\}$ (relative to the inward unit normal) is a constant $\theta_{j} \in\left(-\frac{\pi}{2}, \frac{\pi}{2}\right)$, and is positive iff the direction of reflection has a negative first component in all sufficiently small neighborhoods of 0 . When $\theta_{1}+\theta_{2} \leq 0$, existence and uniqueness in law hold. When $\theta_{1}+\theta_{2}>$ 0 , existence fails. We also obtain results for a large class of asymmetric cusps. We make essential use of results of Warschawski on the differentiability at the boundary of conformal maps.
\end{abstract}

\section{INTRODUCTION AND OUTLINE OF MAIN RESULTS}

In recent years there has been considerable interest in reflecting Brownian motion, abbreviated as RBM, in 2 dimensions. This interest was precipitated by the work of Varadhan and Williams [8] in which they consider an RBM in

$$
D=\left\{z=r e^{i \theta} \in \mathbb{C}: r \geq 0,0 \leq \theta \leq \xi\right\}, \quad \xi \in(0,2 \pi),
$$

with constant oblique angle of reflection on each side of $D$. They gave simple necessary and sufficient conditions for existence and uniqueness. Rogers [3, 4] and Burdzy and Marshall [2] also consider RMB in $D$ but with variable direction of reflection. All of the above mentioned authors explored the conditions under which RBM can reach the origin. Also at issue was extending the process beyond the first time it hits $\{0\}$. All the conditions are of a geometric nature.

Here we study the existence and uniqueness of reflecting Brownian motion in the cusp

$$
C=\left\{(x, y): x \geq 0,-x^{\beta} \leq y \leq x^{\beta}\right\}, \quad \beta>1,
$$

with constant direction of reflection on each of the sides, relative to the inward normals. Because $\partial C \backslash\{0\}$ is smooth, standard results give existence and uniqueness when the process starts away from 0 , up to the first hitting time of 0 .

Received by the editors June 10, 1991.

1991 Mathematics Subject Classification. Primary 60J65; Secondary 60J50.

Key words and phrases. Brownian motion with skew reflection, cusp, conformal transformation, differentiability at the boundary.

Supported in part by grants DMS-8902418 and DMS-8921369 from the National Science Foundation.

Also supported in part by grant DMS-8657483 of Professor R. J. Williams during a visit in August of 1990 to the University of California at San Diego. 
Thus the problem reduces to deciding whether or not $\mathrm{RBM}$ in $C$ starting away from 0 ever hits 0 , and once there can it escape in a unique manner. Hence, in studying existence and uniqueness, the focus of attention should be on the behavior near zero.

Roughly speaking, RBM behaves like Brownian motion inside of $C$, reflects instantly in an oblique direction off the sides and spends zero time at the origin. More precisely, we pose the problem as a question of existence and uniqueness of a solution to a submartingale problem in the style of Stroock and Varadhan [7]. Let $\Omega_{C}$ be the set of continuous functions from $[0, \infty)$ into $C$. For $t \geq 0$ let $\mathscr{M}_{t}$ be the $\sigma$-algebra of subsets of $\Omega_{C}$ generated by the coordinate maps $Z_{s}(\omega)=\omega(s)$ for $0 \leq s \leq t$. We use $\mathscr{M}$ to denote $\sigma\left\{Z_{t}: 0 \leq t<\infty\right\}$. Let $C_{b}^{2}(C)$ be the set of real-valued continuous functions that are defined and twice continuously differentiable on some domain containing $C$ and that together with their first and second partial derivatives are bounded on $C$.

Let $\partial C_{1}=\left\{(x, y) \in C: x \geq 0, y=-x^{\beta}\right\}$ and $\partial C_{2}=\{(x, y) \in C: x \geq$ $\left.0, y=x^{\beta}\right\}$. For $i=1,2$, let $\theta_{i} \in\left(-\frac{\pi}{2}, \frac{\pi}{2}\right)$, for $z \in \partial C_{i} \backslash\{0\}$ let $n_{i}(z)$ be the inward unit normal to $\partial C_{i}$ and let $v_{i}(z)$ make constant angle $\theta_{i}$ with $n_{i}(z)$. We take $\theta_{i}>0$ iff the first component of $v_{i}(z)$ is negative in small neighborhoods of the origin. We also make the normalizations $v_{i}(z) \cdot n_{i}(z)=1$, $z \in \partial C_{i} \backslash\{0\}$.

The Laplacian and gradient will be denoted by $\Delta$ and $\nabla$ as usual. A solution to the submartingale problem on $C$ starting from $z \in C$ is a probability measure $P_{z}$ on $\left(\Omega_{C}, \mathscr{M}\right)$ such that

$$
P_{z}(\omega(0)=z)=1
$$

for each $f \in C_{b}^{2}(C)$,

$$
f(\omega(t))-\frac{1}{2} \int_{0}^{t} \Delta f(\omega(s)) d s
$$

is a $P_{z}$-submartingale on $\left(\Omega_{C}, M,\left\{\mathscr{M}_{t}\right\}\right)$ whenever $f$ is constant in a neighborhood of 0 and $v_{i} \cdot \nabla f \geq 0$ on $\partial C_{i}$ for $i=1,2$;

$$
E^{P_{z}}\left[\int_{0}^{\infty} I_{\{0\}}(\omega(s)) d s\right]=0 .
$$

A family $\left\{P_{z}: z \in C\right\}$ is a solution of the submartingale problem on $C$ if for each $z \in C, P_{z}$ is a solution to the submartingale problem on $C$ starting from $z$. In this case, we say $Z(\cdot)$ together with $\left\{P_{z}: z \in C\right\}$ is an RBM in $C$. The process $Z$ under $P_{z}$ is an RBM in $C$ starting from $z$. Any continuous process having the same law as $Z$ under $P_{z}$ is also called an RBM in $C$ starting from $z$.

Let us briefly describe our method. Let $S=\{(x, y): y \geq 0\}$ be the upper half plane, $\partial S_{1}$ the positive $x$-axis, $\partial S_{2}$ the negative $x$-axis, and let $\{G(t): t \geq 0\}$ be RBM in $S$ with constant angles of reflection $\theta_{1}$ and $\theta_{2}$ on $\partial S_{1}$ and $\partial S_{2}$ respectively. Here $\theta_{1}, \theta_{2} \in\left(-\frac{\pi}{2}, \frac{\pi}{2}\right)$. The sign convention in this case is distinct from that described for $C$ : now the angle of reflection is measured from the inward unit normal and is positive if and only if the associated direction points toward the origin. The process $G$ is shown to exist for all values of $\theta_{1}$ and $\theta_{2}$ in Varadhan and Williams [8]. We will be more precise later. Let $\widetilde{F}: S \rightarrow C$ 
be one-to-one, onto, continuous, and conformal on $S \backslash\{0\}$ with $\widetilde{\mathscr{F}}(0)=0$. It is reasonable to conjecture that if

$$
A(t)=\int_{0}^{t}\left|\widetilde{\mathscr{F}}^{\prime}\left(G_{u}\right)\right|^{2} I\left(G_{u} \neq 0\right) d u<\infty \quad \text { a.s. }
$$

then $\widetilde{Z}(\cdot)=\widetilde{F}\left(G\left(A^{-1}(\cdot)\right)\right)$ ought to have the law of an RBM in $C$ with the desired reflection angles. Hence existence of RBM in $C$ basically comes down to verifying (1.5). Our main result is the following theorem.

Theorem 1.1. There is a unique solution of the submartingale problem on $C$ if $\theta_{1}+\theta_{2} \leq 0$. If $\theta_{1}+\theta_{2}>0$, there is no solution of the submartingale problem on $C$ starting from any $z \in C$.

The reason for nonexistence is that for $\theta_{1}+\theta_{2}>0$, the origin is hit with positive probability and then the process is forced to absorb there, contradicting (1.4). When $\theta_{1}+\theta_{2} \leq 0, G(\cdot)$ started away from 0 will never hit 0 , and hence once $\widetilde{Z}(\cdot)$ is away from 0 it never hits it again. As a by-product of our method we obtain a similar theorem for a large class of asymmetric cusps.

Theorem 1.2. Consider the asymmetric cusp $C=\left\{(x, y): x \geq 0,-x^{\delta} \leq y \leq\right.$ $\left.x^{\beta}\right\}$ where $\beta>1$ and $\delta>2 \beta-1$. There is a unique solution of the submartingale problem on $C$ if $\theta_{1}+\theta_{2} \leq 0$. If $\theta_{1}+\theta_{2}>0$, there is no solution of the submartingale problem on $C$ starting from any $z \in C$.

The question of existence and uniqueness is still open for the case $\delta \in$ $(\beta, 2 \beta-1]$. Unfortunately our method fails in this case. Indeed, it is possible to show that in this case the crucial Theorem 6.2 below is false, by the results of Warschawski [9].

The paper is organized as follows. In $\S 2$ we introduce the conformal transformation $\mathscr{F}$ and various properties are given with the proofs deferred to $\S 6$. In $\S 3$ we develop some preliminaries. Section 4 considers the existence and uniqueness when $\theta_{1}+\theta_{2} \leq 0$ and in $\S 5$ we prove nonexistence for $\theta_{1}+\theta_{2}>0$. Section 7 is devoted to proving Theorem 6.2, a key technical result used in $\S 6$. We prove Theorems 1.1 and 1.2 simultaneously. Hence for the rest of this paper we will assume

$$
\begin{gathered}
C=\left\{(x, y): x \geq 0,-x^{\delta} \leq y \leq x^{\beta}\right\}, \quad \beta>1, \delta=\beta \text { or } \delta>2 \beta-1, \\
\partial C_{1}=\left\{(x, y): x \geq 0, y=-x^{\delta}\right\}, \quad \partial C_{2}=\left\{(x, y): x \geq 0, y=x^{\beta}\right\} .
\end{gathered}
$$

Acknowledgment. We are indebted to Professor Ruth Williams for suggesting this problem to us. Secondly, she told us how to prove Theorem 3.2 and why we needed it in our proof of uniqueness in $\S 4$. Our original proof of nonexistence was rather lengthy and she suggested the method of Varadhan-Williams (1985) should work with much less work. Last but not least we are grateful to her for providing two weeks of support at UCSD for DeBlassie during August of 1990. We are grateful to the referee for the careful review and detailed suggestions for an improved presentation.

\section{Properties of the CONFORMAL TRANSFORMATION}

Since $\partial C \backslash\{0\}$ is smooth, it suffices to construct an RBM in $C$ starting at 0 up to the first time it leaves a small neighborhood of 0 in $C$. Thus we only 
really care about $C$ in a neighborhood of 0 . Hence rather than map $S$ onto $C$, we map a neighborhood of 0 in $S$ onto a neighborhood of 0 in $C$. The next theorem describes properties of a particular choice of such a map. We defer the proof to $\S 6$.

For any $\varepsilon>0$ and $x \in \mathbb{R}^{2}$ let $B_{\varepsilon}(x)=\left\{y \in \mathbb{R}^{2}:|x-y|<\varepsilon\right\}$. For $B \subseteq \mathbb{R}^{2}$ and $n \geq 0$ an integer, let $C^{n}(B)$ denote the set of real-valued continuous functions that are defined and $n$ times continuously differentiable on some domain containing $B$.

Theorem 2.1. There exists an $\varepsilon \in(0,1)$, a closed set $H \subseteq B_{1}(0) \cap C$ and $a$ homeomorphism $\mathscr{F}: S \cap \overline{B_{\varepsilon}(0)} \rightarrow H$ that is conformal on $S \cap \overline{B_{\varepsilon}(0)} \backslash\{0\}$ and has inverse $F$ that is conformal on $H \backslash\{0\}$ such that for some finite constant $K_{1}$,

(i) $F(0)=0$,

(ii) $0 \in \partial H$,

(iii) $H \cap B_{s}(0)=C \cap B_{s}(0)$ for $s$ sufficiently small,

(iv) $\left|\mathscr{F}^{\prime}(\zeta)\right| \leq K_{1}|\zeta|^{-1}(-\ln |\zeta|)^{-\beta /(\beta-1)}, \zeta \in S \cap \overline{B_{\varepsilon}(0)} \backslash\{0\}$,

(v) $\left|F^{\prime}(z)\right| \leq K_{1}, \quad z \in H \backslash\{0\}$,

(vi) $\left|\frac{F^{\prime}(z)}{F(z)}\right|(-\ln |F(z)|)^{-\beta /(\beta-1)} \leq K_{1}, \quad z \in H \backslash\{0\}$.

Moreover, the components of $\mathscr{F}$ are in $C^{2}\left(S \cap \overline{B_{\varepsilon}(0)} \backslash\{0\}\right)$.

\section{Preliminaries}

Let $P_{z}$ be a solution of the submartingale problem on $C$ starting from $z$ and let $Z$ be the canonical process on $\Omega_{C}$. For $0<s<\varepsilon$, define

$$
\sigma_{s}=\inf \{t \geq 0: Z(t) \in\{x \in H:|F(x)|=s\}\} .
$$

Lemma 3.1. If $0<|F(z)|<\varepsilon$ and $\theta_{1}+\theta_{2} \leq 0$ then

$$
P_{z}\left(\sigma_{0} \wedge \sigma_{\varepsilon}<\infty\right)=1 \text {. }
$$

If $\theta_{1}+\theta_{2}>0$ then there exists $\delta_{1} \in(0, \varepsilon]$ such that for $0<|F(z)|<\delta_{1}$,

$$
P_{z}\left(\sigma_{0} \wedge \sigma_{\delta_{1}}<\infty\right)=1 \text {. }
$$

Proof. Define $v_{j}(0)=\lim _{\zeta \rightarrow 0} v_{j}(\zeta)$ along $\partial C_{j} \backslash\{0\}, j=1,2$. The angle $v_{1}(\zeta)$ makes with the positive $x$-axis (the angle measured as positive when taken in the counterclockwise sense) decreases continuously from $\frac{\pi}{2}+\theta_{1}$ towards $\theta_{1}$ as $\zeta$ moves from $\{0\}$ outward along $\partial C_{1}$. Similarly, the angle $v_{2}(\zeta)$ makes with the positive $x$-axis increases continuously from $-\left(\frac{\pi}{2}+\theta_{2}\right)$ towards $-\theta_{2}$ as $\zeta$ moves from $\{0\}$ outward along $\partial C_{2}$.

First consider $\theta_{1}+\theta_{2} \leq 0$. If we regard $v_{j}(\zeta)\left(\zeta \in \partial C_{j}\right)$ as a vector starting at $\{0\}$, then

$$
\begin{aligned}
& \left\{v_{1}(\zeta): \zeta \in \partial C_{1}\right\} \subseteq \bigcup_{r \geq 0}\left\{r e^{i \theta}: \theta_{1} \leq \theta \leq \frac{\pi}{2}+\theta_{1}\right\}=: A_{1}, \\
& \left\{v_{2}(\zeta): \zeta \in \partial C_{2}\right\} \subseteq \bigcup_{r \geq 0}\left\{r e^{i \theta}:-\left(\frac{\pi}{2}+\theta_{2}\right) \leq \theta \leq-\theta_{2}\right\}=: A_{2} .
\end{aligned}
$$

Notice $z_{j} \in A_{j} \Rightarrow v_{j}(\zeta) \cdot z_{j} \geq 0$ for $\zeta \in \partial C_{j}, j=1,2$. Since $\theta_{1}+\theta_{2} \leq 0$, $A_{1} \cap A_{2} \backslash\{0\} \neq \varnothing$. Thus we can choose a unit vector $v \in A_{1} \cap A_{2} \backslash\{0\}$ satisfying

$$
v \cdot v_{j}(\zeta) \geq 0, \quad \zeta \in \partial C_{j}, j=1,2 \text {. }
$$


Next consider $\theta_{1}+\theta_{2}>0$. Then the smallest angle between $v_{1}(0)$ and $v_{2}(0)$ is strictly less than $\pi$. Let $v$ be the unit vector bisecting the angle between $v_{1}(0)$ and $v_{2}(0)$. Then $v \cdot v_{j}(0)>0$ for $j=1,2$. By continuity, for some neighborhood $N$ of 0 in $\mathbb{R}^{2}$ we have $v \cdot v_{j}(\zeta)>0$ for $\zeta \in N \cap \partial C_{j}, j=1,2$. Hence we can choose $\delta_{1} \in(0, \varepsilon]$ so small that

$$
v \cdot v_{j}(\zeta) \geq 0, \quad \zeta \in\left\{w:|F(w)| \leq \delta_{1}\right\} \cap \partial C_{j}, \quad j=1,2 .
$$

This is possible because $\mathscr{F}$ is a homeomorphism.

Now we modify the proof of Lemma 2.1 on p. 411 of Varadhan and Williams [8] as follows. Since $\partial C \backslash\{0\}$ is smooth, for $z \neq 0$ the process $Z\left(\cdot \wedge \sigma_{0}\right)$ has a decomposition under $P_{z}$ :

$$
Z\left(t \wedge \sigma_{0}\right)= \begin{cases}B(t)+\int_{0}^{t} v_{1}(Z(s)) d Y_{1}(s)+\int_{0}^{t} v_{2}(Z(s)) d Y_{2}(s), & 0 \leq t<\sigma_{0}, \\ 0, & t \geq \sigma_{0}\end{cases}
$$

where $Z\left(t \wedge \sigma_{0}\right) \in C$ for all $t \geq 0, B, Y_{1}, Y_{2}$ are adapted to $Z, B\left(\cdot \wedge \sigma_{0}\right)$ is a two-dimensional martingale having mutual variation $\left\langle B_{i}\left(\cdot \wedge \sigma_{0}\right), B_{j}\left(\cdot \wedge \sigma_{0}\right)\right\rangle_{t}=$ $\delta_{i j}\left(t \wedge \sigma_{0}\right)$ for $i, j=1,2$, and $B(0)=z \quad P_{z}$-a.s. $Y_{1}$ and $Y_{2}$ are continuous increasing processes with $Y_{1}(0)=Y_{2}(0)=0$, and $Y_{1}(t), Y_{2}(t)$ are finite for all $t<\sigma_{0}$. Moreover, for each $j=1,2, Y_{j}$ increases only when $Z$ is on $\partial C_{j} \backslash\{0\}$.

Choose $v$ as in (3.2) or (3.3) according to $\theta_{1}+\theta_{2} \leq 0$ or $\theta_{1}+\theta_{2}>0$, and observe that on $\left\{\sigma_{0}=\infty\right\}$ we have for

$$
\begin{gathered}
a=\left\{\begin{array}{ll}
\varepsilon, & \text { if } \theta_{1}+\theta_{2} \leq 0, \\
\delta_{1}, & \text { if } \theta_{1}+\theta_{2}>0
\end{array} \text { and } 0<|F(z)|<a,\right. \\
\sup _{t}\left|Z\left(t \wedge \sigma_{a}\right)\right| \geq \sup _{t}\left[Z\left(t \wedge \sigma_{a}\right) \cdot v\right] \geq \sup _{t}\left[B\left(t \wedge \sigma_{a}\right) \cdot v\right] .
\end{gathered}
$$

Since $\{\zeta \in C:|F(\zeta)| \leq a\} \subseteq\{\zeta \in C:|F(\zeta)| \leq \varepsilon\} \subseteq H \subseteq B_{1}(0) \cap C$ this yields

$$
1 \geq \sup _{t}\left[B\left(t \wedge \sigma_{a}\right) \cdot v\right], \quad \text { a.s. }
$$

By (null) recurrence of the one-dimensional Brownian motion $B(\cdot) \cdot v$,

$$
\sup _{t}[B(t) \cdot v]=+\infty \quad \text { almost surely. }
$$

Hence $\sigma_{a}<\infty$ a.s. on $\left\{\sigma_{0}=\infty\right\}$.

Theorem 3.2. If $0<|F(z)|<\varepsilon$ and $\theta_{1}+\theta_{2} \leq 0$ then $P_{z}\left(\sigma_{0}<\sigma_{\varepsilon}\right)=0$. For $\delta_{1}$ as in Lemma 3.1, if $\theta_{1}+\theta_{2}>0$ then there exists $\delta_{2} \in\left(0, \delta_{1}\right)$ such that

$$
\inf \left\{P_{z}\left(\sigma_{0}<\sigma_{\delta_{1}}\right): 0<|F(z)|<\delta_{2}\right\}>0 .
$$

Proof. Let $\alpha=\left(\theta_{1}+\theta_{2}\right) / \pi$ and in polar coordinates $\zeta=r e^{i \theta}$, set

$$
\Phi(r, \theta)= \begin{cases}r^{\alpha} \cos \left(\alpha \theta-\theta_{1}\right), & \alpha \neq 0, \\ \log r+\theta \tan \theta_{1}, & \alpha=0 .\end{cases}
$$

Then for some constant $K_{2}$ (see Varadhan and Williams [8]-here $V_{i}$ is the direction of reflection on $\partial S_{i}$ making angle $\theta_{i}$ relative to the inward unit 
normal $N_{i}$ to $\partial S_{i}$ with the sign convention on $\theta_{i}$ described in $\S 1$ where $S$ was introduced)

$$
\begin{aligned}
& \Phi \in C^{2}(S \backslash\{0\}), \\
& \Delta \Phi=0 \quad \text { in } S \backslash\{0\}, \\
& V_{i} \cdot \nabla \Phi=0 \text { on } \partial S_{i} \backslash\{0\}, i=1,2, \\
& \Phi(\zeta) \geq K_{2} r^{\alpha} \text { if } \alpha \neq 0 .
\end{aligned}
$$

So if we define $\Psi(\zeta)=\Phi \circ F(\zeta), \zeta \in H$, we see (cf. proof of Proposition 4.1 below) for some positive constants $K_{3}$ and $K_{4}$

$$
\begin{aligned}
& \Psi \in C^{2}(H \backslash\{0\}), \\
& \Delta \Psi=0 \quad \text { in } H \backslash\{0\}, \\
& v_{i} \cdot \nabla \Psi=0 \quad \text { on } \partial C_{i} \cap H \backslash\{0\}, \quad i=1,2, \\
& \Psi(\zeta) \geq K_{3}|F(\zeta)|^{\alpha}, \quad \zeta \in H \backslash\{0\} \quad \text { if } \alpha \neq 0, \\
& \Psi(\zeta) \leq \log |F(\zeta)|+K_{4} \quad \zeta \in H \backslash\{0\} \quad \text { if } \alpha=0 .
\end{aligned}
$$

Thus as in the proof of Theorem 2.2, p. 411 of Varadhan and Williams [8], for $0<s \leq|F(z)| \leq \varepsilon$, by Lemma 3.1, optional stopping and dominated convergence, (recall $\alpha \leq 0$ ),

$$
E_{z}\left[\Psi\left(Z\left(\sigma_{s} \wedge \sigma_{\varepsilon}\right)\right)\right]=\Psi(z) .
$$

Hence for $\alpha<0$ we get

$$
\begin{aligned}
K_{3} s^{\alpha} P_{z}\left(\sigma_{s}<\sigma_{\varepsilon}\right) & \leq E_{z}\left[\Psi\left(Z\left(\sigma_{s}\right)\right) I\left(\sigma_{s}<\sigma_{\varepsilon}\right)\right] \\
& \leq E_{z}\left[\Psi\left(Z\left(\sigma_{s} \wedge \sigma_{\varepsilon}\right)\right)\right]=\Psi(z)
\end{aligned}
$$

or

$$
P_{z}\left(\sigma_{s}<\sigma_{\varepsilon}\right) \leq K_{3}^{-1} s^{-\alpha} \Psi(z) .
$$

By Lemma 3.1, $\sigma_{0} \wedge \sigma_{\varepsilon}<\infty$ a.s. so by continuity of paths, $\left\{\sigma_{0}<\sigma_{\varepsilon}\right\}=$ $\bigcap_{s>0}\left\{\sigma_{s}<\sigma_{\varepsilon}\right\}$ a.s. Hence letting $s \downarrow 0$ in (3.5), $P_{z}\left(\sigma_{0}<\sigma_{\varepsilon}\right)=0$ (since $\alpha<0$ ) as desired.

When $\alpha=0$ and $s$ is small, from (3.4)

$$
\Psi(z)=E_{z}\left[\Psi\left(Z\left(\sigma_{s} \wedge \sigma_{\varepsilon}\right)\right)\right] \leq(\log s) P_{z}\left(\sigma_{s}<\sigma_{\varepsilon}\right)+\log \varepsilon+2 K_{4}
$$

or $($ for $s<1)$

$$
P_{z}\left(\sigma_{s}<\sigma_{\varepsilon}\right) \leq(\log s)^{-1}\left[\Psi(z)-\log \varepsilon-2 K_{4}\right]
$$

and upon letting $s \downarrow 0, P_{z}\left(\sigma_{0}<\sigma_{\varepsilon}\right)=0$ once again.

Finally, we consider $\alpha>0$ (i.e., $\theta_{1}+\theta_{2}>0$ ). Choose $\delta_{3}>0$ such that for $\delta_{1} \in(0, \varepsilon]$ as in Lemma 3.1

$$
\left\{\zeta: \Psi(\zeta) \leq \delta_{3}\right\} \subseteq\left\{\zeta:|F(\zeta)|<\delta_{1}\right\}
$$

This is possible because for some positive $k, \Phi(\zeta) \geq k|\zeta|^{\alpha}, \zeta \in S$. Then choose $\delta_{2}>0$ so that

$$
\delta_{2}<\min \left(\delta,\left(\frac{1}{2} \delta_{3}\right)^{1 / \alpha}\right)
$$

and

$$
\left\{\zeta:|F(\zeta)| \leq \delta_{2}\right\} \subseteq\left\{\zeta: \Psi(\zeta)<\delta_{3}\right\}
$$


The latter is possible because $\alpha>0$ and $\Psi(\zeta) \leq|F(\zeta)|^{\alpha}$ for $\zeta \in H$.

For $r \leq \delta_{3}$ define

$$
\mu_{r}=\inf \left\{t \geq 0: \Psi\left(Z_{t}\right)=r\right\} .
$$

Then $\mu_{0}=\sigma_{0}$ and, as in (3.4) above, for $z$ satisfying $|F(z)|<\delta_{2}$ and $\Psi(z)>$ $s$,

$$
E_{z}\left[\Psi\left(Z\left(\mu_{s} \wedge \mu_{\delta_{3}}\right)\right)\right]=\Psi(z) .
$$

Thus $s P_{z}\left(\mu_{s}<\mu_{\delta_{3}}\right)+\delta_{3}\left(1-P_{z}\left(\mu_{s}<\mu_{\delta_{3}}\right)\right)=\Psi(z)$, which yields

$$
P_{z}\left(\mu_{s}<\mu_{\delta_{3}}\right)=\frac{\Psi(z)-\delta_{3}}{s-\delta_{3}} .
$$

For $z$ satisfying $0<|F(z)|<\delta_{2}$ we have

$$
P_{z}\left(\mu_{0} \wedge \mu_{\delta_{3}}<\infty\right) \geq P_{z}\left(\sigma_{0} \wedge \sigma_{\delta}<\infty\right)=1
$$

by (3.6) and Lemma 3.1. Then by path continuity,

$$
\left\{\mu_{0}<\mu_{\delta_{3}}\right\}=\bigcap_{s>0}\left\{\mu_{s}<\mu_{\delta_{3}}\right\} \text { a.s. } P_{z} .
$$

Hence upon letting $s \downarrow 0$ in (3.9) we get

$$
P_{z}\left(\mu_{0}<\mu_{\delta_{3}}\right)=1-\frac{\Psi(z)}{\delta_{3}}, \quad 0<|F(z)|<\delta_{2} .
$$

But for such $z$, by (3.7) and that $\alpha>0, \Psi(z) \leq|F(z)|^{\alpha}<\delta_{2}^{\alpha}<\frac{1}{2} \delta_{3}$. Hence

$$
\inf \left\{P_{z}\left(\mu_{0}<\mu_{\delta_{3}}\right): 0<|F(z)|<\delta_{2}\right\} \geq \frac{1}{2} \text {. }
$$

To finish, just observe for $0<|F(z)|<\delta_{2}$ we have by (3.6)

$$
P_{z}\left(\sigma_{0}<\sigma_{\delta_{1}}\right) \geq P_{z}\left(\mu_{0}<\mu_{\delta_{3}}\right) .
$$

\section{EXISTENCE AND UNIQUENESS FOR $\theta_{1}+\theta_{2} \leq 0$}

Recall $\beta>1$ and $\delta=\beta$ or $\delta>2 \beta-1$. Throughout this section we assume $\theta_{1}+\theta_{2} \leq 0$. By Theorem 3.2 it suffices to consider an RBM in $C$ starting from 0 . As in the introduction, let $S=\{(x, y): y \geq 0\}$ be the closed upper half-plane. Set $\partial S_{1}=\{(x, 0): x \geq 0\}$ and $\partial S_{2}=\{(x, 0): x \leq 0\}$. Let $N_{j}$ be the unit inward normal to $\partial S_{j}$, and let $V_{j}$ make angle $\theta_{j} \in\left(-\frac{\pi}{2}, \frac{\pi}{2}\right)$ with $N_{j}, j=1,2$. Here $V_{j}$ points toward 0 iff $\theta_{j}>0$. We take $V_{j} \cdot N_{j}=1$. Let $\{G(t): t \geq 0\}$ be a realization of a reflecting Brownian motion (RBM) in $S$ starting at $0 \in S$ with directions of reflection $V_{1}, V_{2}$ on $\partial S_{1} \backslash\{0\}, \partial S_{2} \backslash\{0\}$, respectively. More precisely, on some filtered space $\left(\Omega, \mathscr{S},\left\{\mathscr{S}_{t}\right\}, Q\right)$ there is defined a continuous $\left\{\mathscr{S}_{t}\right\}$-adapted process $G$ such that

(i) $Q\left(G_{u} \in S \forall u \geq 0\right)=1=Q\left(G_{0}=0\right)$;

(ii) for every $f \in C_{b}^{2}(S)$ satisfying $V_{j} \cdot \nabla f \geq 0$ on $\partial S_{j}$ and $f \equiv$ constant near 0 ,

$$
f\left(G_{t}\right)-\int_{0}^{t} \frac{1}{2} \Delta f\left(G_{u}\right) d u \text { is an }\left\{\mathscr{S}_{t}\right\} \text {-submartingale; }
$$

(iii) $E^{Q}\left[\int_{0}^{\infty} I\left(G_{u}=0\right) d u\right]=0$. 
Varadhan and Williams [8] have proved existence and uniqueness in law of $G$ on the space of continuous paths in $S$. For any number $s>0$ set

$$
\eta_{s}=\eta_{s}(G)=\inf \left\{t>0:\left|G_{t}\right|=s\right\}
$$

and define (here $\varepsilon$ is from Theorem 2.1)

$$
A^{G}(t)=\int_{0}^{t \wedge \eta_{\varepsilon}}\left|\mathscr{F}^{\prime}\left(G_{u}\right)\right|^{2} I\left(G_{u} \neq 0\right) d u
$$

If

$$
A^{G}\left(\eta_{\varepsilon}(G)\right)<\infty
$$

then $A^{G}$ is continuous (hence finite) on $\left[0, \eta_{\varepsilon}\right]$. Since $\mathscr{F}$ is conformal away from 0 (so $\mathscr{F}^{\prime} \neq 0$ there) and $G$ does not spend positive time at 0 a.s. $(Q)$, under (4.2) $A^{G}$ is strictly increasing on $\left[0, \eta_{\varepsilon}\right]$. Then $A^{G}$ on $\left[0, \eta_{\varepsilon}\right]$ has a strictly increasing continuous inverse $a_{t}^{G}$ defined on $\left[0, A^{G}\left(\eta_{\varepsilon}\right)\right]$. Define

$$
Z^{G}(t)=\mathscr{F}\left(G\left(a^{G}\left(t \wedge A^{G}\left(\eta_{\varepsilon}\right)\right)\right)\right), \quad \text { for } t \geq 0 .
$$

We will sometimes drop the superscript $G$ in the notation.

Recall that we need only construct an RBM starting from 0 until it leaves a small neighborhood of 0 in $C$. Thus for existence we need only verify (1.2)(1.4) for the law of $Z^{G}$ on $\left(\Omega_{C}, \mathscr{M}\right)$ with $t$ replaced by $t \wedge \sigma_{\varepsilon}$ where

$$
\sigma_{\varepsilon}(\omega)=\sigma_{\varepsilon}=\inf \left\{t>0:\left|F\left(\omega_{t}\right)\right|=\varepsilon\right\} .
$$

If this is true, we say $Z^{G}(\cdot)$ is an $R B M$ in $C$, stopped at time $\sigma_{\varepsilon}$, starting at 0 . We will usually not include the statement "starting at 0 ".

Proposition 4.1. If $A\left(\eta_{\varepsilon}\right)<\infty$ then $Z^{G}(\cdot)$ given by (4.3) is an $R B M$ in $C$ stopped at time $\sigma_{\varepsilon}$.

Proof. Since $F(0)=0$,

$$
E^{Q}\left[I\left(Z_{0}^{G}=0\right)\right]=E^{Q}\left[I\left(G_{0}=0\right)\right]=1 .
$$

Also, since $F(H)=S \cap \overline{B_{\varepsilon}(0)}, \sigma_{\varepsilon}=A\left(\eta_{\varepsilon}\right)$ and so

$$
\begin{aligned}
E^{Q}\left[\int_{0}^{\sigma_{\varepsilon}} I\left(Z_{s}^{G}=0\right) d s\right] & =E^{Q}\left[\int_{0}^{\sigma_{\varepsilon}} I\left(\mathscr{F}\left(G\left(a_{s}\right)\right)=0\right) d s\right] \\
& =E^{Q}\left[\int_{0}^{\sigma_{\varepsilon}} I\left(G\left(a_{s}\right)=0\right) d s\right] \\
& =0
\end{aligned}
$$

since $a(\cdot)$ is strictly increasing and $E^{Q}\left[\int_{0}^{\infty} I\left(G_{s}=0\right) d s\right]=0$.

To show (1.3) suppose $f \in C_{b}^{2}(C)$ is constant in a neighborhood of 0 and $v_{i} \cdot \nabla f \geq 0$ on $\partial C_{i}, i=1,2$. Let $h(\zeta)=f \circ \mathscr{F}(\zeta), \zeta \in S \cap \overline{B_{\varepsilon}(0)}$. Then $h$ is constant in a neighborhood of 0 and $h \in C_{b}^{2}\left(S \cap \overline{B_{\varepsilon}(0)}\right)$.

Let

$$
J(z)=\left(\begin{array}{ll}
\frac{\partial F_{1}}{\partial z_{1}} & \frac{\partial F_{1}}{\partial z_{2}} \\
\frac{\partial F_{2}}{\partial z_{1}} & \frac{\partial F_{2}}{\partial z_{2}}
\end{array}\right), \quad z=\left(z_{1}, z_{2}\right),
$$


be the Jacobian of $F$. By conformality, for some positive $c_{j}(z)$,

$$
V_{j}=c_{j}(z) J(z) v_{j}(z), \quad z \in\left(\partial C_{j} \backslash\{0\}\right) \cap H, j=1,2 .
$$

Also, for $\zeta=F(z)$ and $\mathscr{J}(\zeta)$ the Jacobian of $\mathscr{F}$ at $\zeta$,

$$
\nabla h(\zeta)=\nabla(f \circ \mathscr{F})(\zeta)=\mathscr{J}(\zeta)^{*}(\nabla f)(\mathscr{F}(\zeta))=\left(J^{-1}(z)\right)^{*}(\nabla f)(z) .
$$

Thus for $\zeta \in\left(\partial S_{j} \backslash\{0\}\right) \cap \overline{B_{\varepsilon}(0)}$, we have $z=\mathscr{F}(\zeta) \in\left(\partial C_{j} \backslash\{0\}\right) \cap H$ and

$$
\begin{aligned}
\nabla h(\zeta) \cdot V_{j} & =(\nabla h(\zeta))^{*} V_{j} \quad(\text { as matrices }) \\
& =\left[((\nabla f)(z))^{*} J^{-1}(z)\right] c_{j}(z) J(z) v_{j}(z) \\
& =c_{j}(z) \nabla f(z) \cdot v_{j}(z) \geq 0 .
\end{aligned}
$$

Thus

$$
\begin{aligned}
& f\left(Z_{t \wedge \sigma_{\varepsilon}}^{G}\right)-\frac{1}{2} \int_{0}^{t \wedge \sigma_{\varepsilon}} \Delta f\left(Z_{s}^{G}\right) d s \\
& =h\left(G_{a\left(t \wedge \sigma_{\varepsilon}\right)}\right)-\frac{1}{2} \int_{0}^{t \wedge \sigma_{\varepsilon}} \Delta(h \circ F)\left(\mathscr{F}\left(G_{a(s)}\right)\right) d s \\
& =h\left(G_{a\left(t \wedge \sigma_{\varepsilon}\right)}\right)-\frac{1}{2} \int_{0}^{t \wedge \sigma_{\varepsilon}} \Delta h\left(G_{a(s)}\right)\left|F^{\prime}\left(\mathscr{F}\left(G_{a(s)}\right)\right)\right|^{2} d s \\
& =h\left(G_{a\left(t \wedge \sigma_{\varepsilon}\right)}\right)-\frac{1}{2} \int_{0}^{a\left(t \wedge \sigma_{\varepsilon}\right)} \Delta h\left(G_{u}\right) I\left(G_{u} \neq 0\right) d u \\
& =h\left(G_{a\left(t \wedge \sigma_{\varepsilon}\right)}\right)-\frac{1}{2} \int_{0}^{a\left(t \wedge \sigma_{\varepsilon}\right)} \Delta h\left(G_{s}\right) d s
\end{aligned}
$$

is a submartingale.

We can reverse the preceding procedure. Indeed, given a realization $Z(\cdot)$ of RBM in $C$ stopped at time $\sigma_{\varepsilon}(Z)$ starting from 0 , since $\theta_{1}+\theta_{2} \leq 0$, Lemma 3.1 and Theorem 3.2 imply $\sigma_{\varepsilon}(Z)<\infty$ a.s. Set

$$
V^{Z}(t)=\int_{0}^{t \wedge \sigma_{\varepsilon}(Z)}\left|F^{\prime}\left(Z_{u}\right)\right|^{2} I\left(Z_{u} \neq 0\right) d u, \quad t \geq 0 .
$$

By Theorem 2.1, for some constant $K, V^{Z}(t) \leq K t$ for all $t \geq 0$ and $V^{Z}$ is continuous and strictly increasing on $\left[0, \sigma_{\varepsilon}(Z)\right]$. Hence it has a continuous strictly increasing inverse $v^{Z}(t)$ on $\left[0, V^{Z}\left(\sigma_{\varepsilon}(Z)\right)\right]$. Define

$$
G^{Z}(t)=F\left(Z\left(v^{Z}\left(t \wedge V^{Z}\left(\sigma_{\varepsilon}(Z)\right)\right)\right)\right), \quad t \geq 0 .
$$

Similar to Proposition 4.1, $G^{Z}$ is an RBM in $S$ stopped at $V^{Z}\left(\sigma_{\varepsilon}(Z)\right)$.

We now use the above to show existence and uniqueness of RBM in $C$ stopped at $\sigma_{\varepsilon}(Z)$.

Theorem 4.2. Let $G$ be an RBM in $S$ starting at 0 . If $\theta_{1}+\theta_{2} \leq 0$ then the condition

$$
\int_{0}^{\eta_{\ell}(G)}\left|G_{u}\right|^{-2}|\ln | G_{u}||^{-\frac{2 \beta}{\beta-1}} I\left(G_{u} \neq 0\right) d u<\infty
$$

is sufficient for existence and uniqueness in law of RBM $Z$ in $C$ stopped at time $\sigma_{\varepsilon}(Z)$.

Proof. Assuming (4.6) holds, then by Theorem 2.1(iv), $A^{G}\left(\eta_{\varepsilon}(G)\right)<\infty$. Hence existence follows from Proposition 4.1. 
Next w.e consider uniqueness. Observe

$$
\left\{\begin{array}{l}
V^{Z}\left(\sigma_{\varepsilon}(Z)\right)=\eta_{\varepsilon}\left(G^{Z}\right) \\
A^{G}\left(\eta_{\varepsilon}(G)\right)=\sigma_{\varepsilon}\left(Z^{G}\right) .
\end{array}\right.
$$

Then since the law of RBM on $S$ stopped at $\eta_{\varepsilon}$ is unique, we need only show that the associations

$$
\begin{aligned}
& Z\left(\cdot \wedge \sigma_{\varepsilon}(Z)\right) \mathrm{RBM} \text { in } C \text { stopped at } \sigma_{\varepsilon}(Z) \\
& \quad \rightarrow G^{Z}\left(\cdot \wedge V^{Z}\left(\sigma_{\varepsilon}(Z)\right)\right) \mathrm{RBM} \text { in } S \text { stopped at } \eta_{\varepsilon}\left(G^{Z}\right)
\end{aligned}
$$

and

$$
\begin{aligned}
G(\cdot \wedge & \left.\sigma_{\varepsilon}(G)\right) \mathrm{RBM} \text { in } S \text { stopped at } \eta_{\varepsilon}(Z) \\
& \rightarrow Z^{G}\left(\cdot \wedge A^{G}\left(\eta_{\varepsilon}(G)\right)\right) \mathrm{RBM} \text { in } C \text { stopped at } \sigma_{\varepsilon}\left(Z^{G}\right)
\end{aligned}
$$

form a one-to-one correspondence. For this it suffices to show

$$
\sigma_{\varepsilon}\left(Z^{\left(G^{Z}\right)}\right)=\sigma_{\varepsilon}(Z), \quad Z^{\left(G^{Z}\right)}(\cdot)=Z\left(\cdot \wedge \sigma_{\varepsilon}(Z)\right),
$$

and

$$
\eta_{\varepsilon}\left(G^{\left(Z^{G}\right)}\right)=\eta_{\varepsilon}(G), \quad G^{\left(Z^{G}\right)}(\cdot)=G\left(\cdot \wedge \eta_{\varepsilon}(G)\right) .
$$

We only prove (4.8), the proof of (4.9) being similar.

Observe first that

$$
A^{\left(G^{Z}\right)}\left(V^{Z}(t)\right)=t, \quad t \leq \sigma_{\varepsilon}(Z) .
$$

Indeed, for such $t, V^{Z}(t) \leq V^{Z}\left(\sigma_{\varepsilon}(Z)\right)=\eta_{\varepsilon}\left(G^{Z}\right)$ (by (4.7)) so the left side is well defined and by $(4.1)$ is

$$
\begin{aligned}
\int_{0}^{V^{Z}(t)} & \left|\mathscr{F}^{\prime}\left(G_{u}^{Z}\right)\right|^{2} I\left(G_{u}^{Z} \neq 0\right) d u \\
\quad= & \int_{0}^{V^{Z}(t)}\left|\mathscr{F}^{\prime} \circ F\left(Z\left(v_{u}^{Z}\right)\right)\right|^{2} I\left(Z\left(v_{u}^{Z}\right) \neq 0\right) d u \quad(\text { by }(4.5)) \\
& =\int_{0}^{t}\left|\mathscr{F}^{\prime} \circ F\left(Z_{s}\right)\right|^{2} I\left(Z_{s} \neq 0\right)\left|F^{\prime}\left(Z_{s}\right)\right|^{2} d s \quad(\text { by }(4.4)) \\
& =\int_{0}^{t} I\left(Z_{s} \neq 0\right) d s \\
& =t .
\end{aligned}
$$

Applying this, by (4.7),

$$
\sigma_{\varepsilon}\left(Z^{\left(G^{Z}\right)}\right)=A^{\left(G^{Z}\right)}\left(\eta_{\varepsilon}\left(G^{Z}\right)\right)=A^{\left(G^{Z}\right)}\left(V^{Z}\left(\sigma_{\varepsilon}(Z)\right)\right)=\sigma_{\varepsilon}(Z),
$$

giving the first part of (4.8).

For the latter part, by (4.10), $v^{Z}\left(a^{\left(G^{Z}\right)}(t)\right)=t$ for $t \leq \sigma_{\varepsilon}(Z)$ and so

$$
\begin{aligned}
Z^{\left(G^{Z}\right)}(t) & =\mathscr{F}\left(G^{Z}\left\{a^{G^{Z}}\left(t \wedge A^{G^{Z}}\left(\eta_{\varepsilon}\left(G^{Z}\right)\right)\right)\right\}\right) \quad \text { by }(4.3) \\
& =\mathscr{F}\left(G^{Z}\left\{a^{G^{Z}}\left(t \wedge \sigma_{\varepsilon}\left(Z^{G^{Z}}\right)\right)\right\}\right) \quad \text { by }(4.7) \\
& =\mathscr{F}\left(G^{Z}\left\{a^{G^{Z}}\left(t \wedge \sigma_{\varepsilon}(Z)\right)\right\}\right) \quad(\text { first part of }(4.8)) \\
& =Z\left(v^{Z}\left\{\left[a^{G^{Z}}\left(t \wedge \sigma_{\varepsilon}(Z)\right)\right] \wedge V^{Z}\left(\sigma_{\varepsilon}(Z)\right)\right\}\right) \quad \text { by }(4.5) \\
& =Z\left(t \wedge \sigma_{\varepsilon}(Z)\right)
\end{aligned}
$$

as desired. 
By Theorem 4.2, to prove existence and uniqueness of RBM for $\theta_{1}+\theta_{2} \leq 0$, it suffices to verify (4.6). For this we need the following theorem.

Theorem 4.3. Let $h \in C\left(S \cap \overline{B_{\varepsilon}(0)}\right) \cap C^{2}\left(S \cap \overline{B_{\varepsilon}\{0\}} \backslash\{0\}\right)$ with $h(0)=0, \Delta h \geq 0$ on $S \backslash\{0\}$, and $V_{j} \cdot \nabla h \geq 0$ on $\left(\partial S_{j}\right) \cap \overline{B_{\varepsilon}(0)} \backslash\{0\}, j=1,2$. Then

$$
E^{Q}\left[\int_{0}^{t \wedge \eta_{e}} \Delta h\left(G_{s}\right) I_{\left\{h\left(G_{s}\right)>0\right\}} d s\right]<\infty .
$$

Proof. Suppose $g_{n}: \mathbb{R} \rightarrow[0, \infty)$ is continuous with $\operatorname{supp} g_{n} \subseteq\left[\frac{1}{3 n}, \frac{1}{n}\right]$ and $\int g_{n}(r) d r=1$. Let

$$
k_{n}(t)=\int_{0}^{t} \int_{0}^{s} g_{n}(r) d r d s .
$$

Then $k_{n} \in C^{2}(\mathbb{R}), k_{n}$ vanishes in a neighborhood of 0 and $\left|k_{n}(t)-t \vee 0\right| \leq \frac{A}{n}$ for some constant $A>0$. Moreover, $0 \leq k_{n}^{\prime} \leq 1, k_{n}^{\prime \prime} \geq 0$ and as $n \rightarrow \infty$, $k_{n}^{\prime}(t) \rightarrow I_{(0, \infty)}(t)$.

With $h$ as above, it follows that $k_{n} \circ h \in C_{b}^{2}\left(S \cap \overline{B_{\varepsilon}(0)}\right)$. Now $k_{n} \circ h$ can be extended outside of $S \cap \overline{B_{\varepsilon}(0)}$ so that it is in $C_{b}^{2}(S)$ and satisfies $V_{j} \cdot \nabla\left(k_{n} \circ h\right) \geq 0$ on $\partial S_{j}, j=1,2$. Such extensions are made in Varadhan and Williams [8]. Therefore by the submartingale property

$$
\begin{aligned}
& E^{Q}\left[k_{n} \circ h\left(G_{t \wedge \eta_{e}}\right)-k_{n} \circ h(z)\right] \\
& \quad \geq \frac{1}{2} E^{Q}\left[\int_{0}^{t \wedge \eta_{e}}\left[\Delta h\left(G_{s}\right) k_{n}^{\prime} \circ h\left(G_{s}\right)+\left|\nabla h\left(G_{s}\right)\right|^{2} k_{n}^{\prime \prime} \circ h\left(G_{s}\right)\right] d s\right] .
\end{aligned}
$$

Note that since $h$ is bounded on $S \cap \overline{B_{\varepsilon}(0)}$, as $n \rightarrow \infty$ the left hand side converges to $E^{Q}\left[0 \vee h\left(G_{t \wedge \eta_{e}}\right)\right]-0 \vee h(z)$. By hypothesis, all integrands on the right are nonnegative. Hence by Fatou's lemma, since $k_{n}^{\prime}$ converges to $I_{(0, \infty)}$,

$$
E^{Q}\left[\int_{0}^{t \wedge \eta_{\varepsilon}} \Delta h\left(G_{s}\right) I\left(h\left(G_{s}\right)>0\right) d s\right]<\infty
$$

is desired.

Now we specialize this to verify (4.6). In polar coordinates $(r, \theta)$ for $z=$ $r e^{i \theta}$ let

$$
h(z)= \begin{cases}{\left[-\ln r-\theta \tan \theta_{1}\right]^{-\frac{2}{\beta-1}},} & z \in \overline{B_{\varepsilon}(0)} \cap S \backslash\{0\}, \\ 0, & r=0,\end{cases}
$$

where $\beta$ is the same number used to define the cusp $C$. By making $\varepsilon$ from Theorem 2.1 smaller if necessary, for $r \leq \varepsilon$ we have $h \geq 0, h(0)=0, h \in$ $C\left(S \cap \overline{B_{\varepsilon}(0)}\right) \cap C^{2}\left(S \cap \overline{B_{\varepsilon}(0)} \backslash\{0\}\right)$ and

$$
\Delta h=\frac{2}{\beta-1} r^{-2}\left[-\ln r-\theta \tan \theta_{1}\right]^{-\frac{2 \beta}{\beta-1}}\left(\frac{2}{\beta-1}+1\right)\left(1+\tan ^{2} \theta_{1}\right) .
$$

Then $\Delta h \geq 0$ on $S \cap \overline{B_{\varepsilon}(0)} \backslash\{0\}$ and $\Delta h \geq K r^{-2}[-\ln r]^{-\frac{2 \beta}{\beta-1}}$ for $0<r \leq \varepsilon$ and some constant $K>0$ depending on $\varepsilon, \beta$ and $\tan \left|\theta_{1}\right|$. In polar coordinates $\nabla h=\left(\frac{\partial h}{\partial r}, \frac{1}{r} \frac{\partial h}{\partial \theta}\right)$ so

$$
\nabla h=\frac{2}{\beta-1}\left(-\ln r-\theta \tan \theta_{1}\right)^{-\frac{2}{\beta-1}-1}\left(\frac{1}{r}, \frac{1}{r} \tan \theta_{1}\right) ;
$$


also,

$$
V_{1}=\left(-\tan \theta_{1}, 1\right) \text { and } V_{2}=\left(-\tan \theta_{2},-1\right) .
$$

It is easily verified that $V_{j} \cdot \nabla h \geq 0$ on $\left(\partial S_{j} \backslash\{0\}\right) \cap \overline{B_{\varepsilon}(0)}, j=1,2$, because $\theta_{1}+\theta_{2} \leq 0$. Therefore $h$ satisfies the conditions of Theorem 4.3 on $\overline{B_{\varepsilon}(0)} \cap S$ and

$$
\begin{aligned}
& E^{Q}\left[\int_{0}^{t \wedge \eta_{\varepsilon}}\left|G_{s}\right|^{-2}|\ln | G_{s}||^{-\frac{2 \beta}{\beta-1}} I_{\left\{G_{s} \neq 0\right\}} d s\right] \\
& \quad \leq K^{-1} E^{Q}\left[\int_{0}^{t \wedge \eta_{\varepsilon}} \Delta h\left(G_{s}\right) I_{\left\{h\left(G_{s}\right)>0\right\}} d s\right]<\infty
\end{aligned}
$$

as desired.

Remark. If $\theta_{1}+\theta_{2} \leq 0$ then by Lemma 3.1 and Theorem 3.2 an RBM in $C$ starting away from 0 never hits 0 . Of course, the preceding calculations show the process can be started from 0 .

\section{RBM IN $C$ DOES NOT EXIST IF $\theta_{1}+\theta_{2}>0$}

Throughout this section we take $\theta_{1}+\theta_{2}>0$.

Theorem 5.1. Suppose $\theta_{1}+\theta_{2}>0$. Then for any $z \in C$, there is no solution to the submartingale problem on $C$ starting from $z$.

First we prove the following special case.

Theorem 5.2. Suppose $\theta_{1}+\theta_{2}>0$. Then there exists no solution to the submartingale problem on $C$ starting from 0 .

Proof. Suppose there exists a process $Z$ with law $P_{0}$ on $\left(\Omega_{C}, \mathscr{M}\right)$ satisfying (1.2) and (1.3). We will show below that (1.4) cannot also hold for this process and therefore an RBM in $C$ starting from 0 does not exist when $\theta_{1}+\theta_{2}>0$.

Let $\alpha, \Phi(r, \theta)$ and $\Psi(Z)$ be defined as in the proof of Theorem 3.2. Let $\varepsilon$ be as in Theorem 2.1 and set $c=\cos \theta_{1} \wedge \cos \theta_{2}$; note $c>0$. Define $K=c(\varepsilon / 2)^{\alpha}$ and let

$$
\mu_{K}=\inf \left\{t>0: \Psi\left(Z_{t}\right)=K\right\} .
$$

We will prove below that

$$
P_{0}\left[w\left(t \wedge \mu_{K}\right)=0 \text { for all } t\right]=1 .
$$

Because $\Phi(r, \theta)>0$ for $(r, \theta) \in S \cap B_{\varepsilon}(0) \backslash\{0\}$ and $\Phi(0, \theta)=0$ when $\theta_{1}+\theta_{2}>0$, by continuity of $\Phi$, there exists an open neighborhood $J$ of $\{0\}$ in $H$ for which $\Psi(z)<K$ for all $z \in J$. Therefore continuity of paths and (1.2) imply $\mu_{K}>0$ a.s. so (5.1) and (1.4) cannot both hold. that

Let $g:[0, \infty) \rightarrow[0,1]$ be a twice continuously differentiable function such

$$
g(x)= \begin{cases}0, & 0 \leq x \leq 1 / 2 \\ 1, & x \geq 1\end{cases}
$$

and let $M=\max _{x \in[0, \infty)}\left\{\left|g^{\prime}(x)\right|+\left|g^{\prime \prime}(x)\right|\right\}$. For each $s>0$ with $s \leq K$ and $z \in H$ define

$$
\begin{aligned}
& h_{s}(z)=g\left(s^{-1} \Psi(z)\right) ; \\
& f_{s}(z)= \begin{cases}h_{s}(z)\left(1-h_{2 K}(z)\right) \Psi(z), & 2 K \geq \Psi(z) \geq s / 4, \\
0, & \text { otherwise. }\end{cases}
\end{aligned}
$$


Because $F$ is conformal in $H \backslash\{0\}, f_{s}$ is well defined as an element of $C_{b}^{2}(H)$, constant in a neighborhood of $\{0\}$ and $\nabla f_{s} \cdot v_{j}=0$ on $\overline{B_{\varepsilon}(0)} \cap \partial C_{j}$. Moreover $\Delta \Psi=((\Delta \Phi)(F))\left|F^{\prime}\right|^{2}$ on $H \backslash\{0\}$ and since $\Delta \Phi=0$ in $S \backslash\{0\}$ it is easy to show for $z \in H$,

$$
\Delta f_{s}(z)= \begin{cases}0, & 0 \leq \Psi \leq s / 2, \\ \left(s^{-2} g^{\prime \prime} \Psi+2 s^{-1} g^{\prime}\right)|(\nabla \Phi)(F)|^{2}\left|F^{\prime}\right|^{2}(z), & s / 2 \leq \Psi \leq s, \\ 0, & s \leq \Psi \leq K\end{cases}
$$

By Doob's stopping theorem and (1.3), with equality,

$$
f_{s}\left(w\left(t \wedge \mu_{K}\right)\right)-\frac{1}{2} \int_{0}^{t \wedge \mu_{K}} \Delta f_{s}(w(u)) d u
$$

is a $P^{0}$ martingale. Also on $\{z \in C: 0 \leq \Psi(z) \leq K\}, f_{s} \rightarrow \Psi$ uniformly and $\Delta f_{s} \rightarrow 0$ as $s \downarrow 0$. By (2.31) of Varadhan and Williams [8] there is a constant $b<\infty$ such that $|(\nabla \Phi)(F)|^{2} \leq b \Psi^{2-2 / \alpha}$ on $H \backslash\{0\}$. By Theorem 2.1vi), on $H \backslash\{0\}$,

$$
\left|F^{\prime}(z)\right| \leq K_{1}|F(z)|(-\ln |F(z)|)^{\beta /(\beta-1)} .
$$

But on $\{z: s / 2 \leq \Psi \leq s\},|F(z)| \leq\left(\frac{s}{c}\right)^{1 / \alpha}$ so for $|z|$ sufficiently small, since $x(-\ln x)^{\beta /(\beta-1)}$ is an increasing function for small positive $x$,

$$
\left|F^{\prime}(z)\right|^{2} \leq K_{1}^{2}\left(\frac{s}{c}\right)^{2 / \alpha}\left(-\frac{1}{\alpha} \ln \left|\frac{s}{c}\right|\right)^{2 \beta /(\beta-1)} .
$$

Thus on $\{z \in C: 0 \leq \Psi(z) \leq K\}$,

$$
\begin{aligned}
\left|\Delta f_{s}\right| & \leq K_{5}\left\{\left(s^{-2} M\right) s+2 s^{-1} M\right\} s^{2-2 / \alpha} s^{2 / \alpha}\left(-\frac{1}{\alpha} \ln \left|\frac{s}{c}\right|\right)^{2 \beta /(\beta-1)} \\
& =K_{6} s\left(-\frac{1}{\alpha} \ln \left|\frac{s}{c}\right|\right)^{2 \beta /(\beta-1)}
\end{aligned}
$$

which is bounded uniformly in $s$ on $\{z \in C: 0 \leq \Psi(z) \leq K\}$. Because martingales are preserved under bounded convergence at each time $t$, by letting $s \downarrow 0$ in (5.2) we find $\Psi\left(w\left(t \wedge \mu_{K}\right)\right)$ is a $P_{0}$ martingale. Hence

$$
E_{0}\left[\Psi\left(w\left(t \wedge \mu_{K}\right)\right)\right]=\Psi(0)=0
$$

for all $t \geq 0$. Since $\Psi>0$ on $C \backslash\{0\}$ this implies (5.1) and we are done.

On $\Omega_{C}$, for $Z(\cdot)$ the coordinate process and $r \geq 0$, define

$$
\tau_{r}=\tau_{r}(Z)=\inf \{t \geq 0:|Z(t)|=r\} .
$$

The proof of the following theorem is much like that of Theorem 2.1 on p. 410 of Varadhan-Williams [8].

Theorem 5.3. For each $z \in C$ there is a unique probability measure $P_{z}^{0}$ on $\Omega_{C}$ such that

(i) $P_{z}^{0}(Z(0)=z)=1$;

(ii) for each $f \in C_{b}^{2}(C)$ satisfying $v_{j} \cdot \nabla f \geq 0$ on $\partial C_{j}, j=1,2$,

$$
f\left(Z\left(t \wedge \tau_{0}\right)\right)-\frac{1}{2} \int_{0}^{t \wedge \tau_{0}}(\Delta f)(Z(s)) d s
$$

is a $P_{z}^{0}$-submartingale;

(iii) $P_{z}^{0}\left(Z(t)=0 \forall t \geq \tau_{0}\right)=1$ 
Moreover, the family $\left\{P_{z}^{0}: z \in C\right\}$ has the strong Markov property.

In light of Theorem 5.2, Theorem 5.1 is a consequence of the following result.

Theorem 5.4. For $z \in C \backslash\{0\}, P_{z}^{0}\left(\tau_{0}<\infty\right)>0$.

Proof. Let $\delta_{1}$ and $\delta_{2}$ be from Lemma 3.1 and Theorem 3.2, respectively. Thus $0<\delta_{2}<\delta_{1} \leq \varepsilon$. If $z$ satisfies $0<|F(z)|<\delta_{2}$, then by Theorem 3.2

$$
0<P_{z}\left(\sigma_{0}<\sigma_{\delta_{1}}\right)=P_{z}^{0}\left(\sigma_{0}<\sigma_{\delta_{1}}\right) \leq P_{z}^{0}\left(\tau_{0}<\infty\right),
$$

as desired. Define $B=\left\{\zeta \in C: 0<|F(\zeta)|<\frac{1}{2} \delta_{2}\right\}$ and $\tau_{B}=\inf \{t \geq 0$ : $\left.Z_{t} \in B\right\}$. Then for each $z \in C \backslash\{0\}, P_{z}^{0}\left(\tau_{B}<\infty\right)>0$. Indeed, for $z$ in the interior of $C$, this follows from properties of ordinary Brownian motion in $C$; for $z$ in the boundary of $\partial C$ excluding the origin, reflecting Brownian motion will enter the interior of $C$ immediately and from there will have positive probability of hitting $B$. Together with (5.3) and the strong Markov property, this yields the desired conclusion for $z$ satisfying $|F(z)| \geq \delta_{2}$.

\section{Proof of Theorem 2.1}

Our plan of action is to define a function $F$ explicitly enough to prove it has the properties stated in Theorem 2.1. In essence we do the following. Open the cusp via a conformal mapping to locally flatten the boundary near 0 so that the tangent is well defined there. Then conformally map to the unit disc; from there map conformally to the upper half-space $S$. This seems convoluted because Warschawski [9] does have results on conformal maps from a cusp to the unit disc. The trouble is, the results are too coarse for our purpose. On the other hand, he has results on conformal maps from domains with continuously turning tangents to the unit disc and they are good enough for us.

First we open the cusp. Below in (7.1) we give an explicit parametric representation of $\partial C_{1}$ and $\partial C_{2}$ in polar coordinates. With this in mind, using the principal branch of $\ln z$, set

$$
f_{\delta}(z)= \begin{cases}0, & z=0, \\ \exp \left\{-\frac{\pi}{2(\beta-1)} \exp \{-(\beta-1) \ln z\}\right\}, & z \neq 0, \delta=\beta \\ \exp \left\{-\frac{\pi}{(\beta-1)} \exp \{-(\beta-1) \ln z\}-i \frac{\pi}{2}\right\}, & z \neq 0, \delta>\beta\end{cases}
$$

We will show below $\left((7.2)-(7.3)\right.$ and Lemma 7.3) that $f_{\delta}$ does indeed open the cusp. Now for $\gamma \in(0,1)$ sufficiently small, $f$ is well defined and one-to-one on $N=\{z:|z| \leq \gamma, \operatorname{Re} z \geq 0\} \cap C$. Moreover, $f_{\delta}$ and $f_{\delta}^{-1}$, are conformal on $N \backslash\{0\}$ and $f(N \backslash\{0\})$, respectively, with

$$
f_{\delta}^{-1}(w)= \begin{cases}0, & w=0, \\ \exp \left\{-\frac{1}{\beta-1} \ln \left(-\frac{2(\beta-1)}{\pi} \ln w\right)\right\}, & w \neq 0, \delta=\beta \\ \exp \left\{-\frac{1}{\beta-1} \ln \left(-\frac{\beta-1}{\pi}\left[\ln w+i \frac{\pi}{2}\right]\right)\right\}, & w \neq 0, \delta>\beta\end{cases}
$$

Notice

$$
f_{\delta}(z)=e^{-i \pi / 2}\left[f_{\beta}(z)\right]^{2} \quad \text { for } \delta>\beta .
$$


In polar coordinates $z=r e^{i \theta}, \theta \in\left[-\frac{\pi}{2}, \frac{\pi}{2}\right]$,

$$
\left\{\begin{array}{l}
f_{\beta}(z)=\exp \left\{-\frac{\pi}{2(\beta-1)} r^{-(\beta-1)} \cos [(\beta-1) \theta]\right\} e^{i \varphi_{\beta}}, \\
f_{\delta}(z)=\left|f_{\beta}(z)\right|^{2} e^{i \varphi_{\delta}}, \quad \delta>\beta,
\end{array}\right.
$$

where

$$
\left\{\begin{array}{l}
\varphi_{\beta}=\frac{\pi}{2(\beta-1)} r^{-(\beta-1)} \sin [(\beta-1) \theta], \\
\varphi_{\delta}=2 \varphi_{\beta}-\frac{\pi}{2}, \quad \delta>\beta
\end{array}\right.
$$

We leave the proof of the following elementary lemma to the reader.

Lemma 6.1. The following bounds hold

$$
\begin{gathered}
\left|f_{\delta}^{\prime}(z)\right| \leq C\left|f_{\delta}(z)\right||z|^{-\beta}, \quad z \in N \backslash\{0\}, \\
\left|\left(f_{\delta}^{-1}\right)^{\prime}(w)\right| \leq C|w|^{-1}(-\ln |w|)^{-\frac{\beta}{\beta-1}}, \quad w \in f(N \backslash\{0\}) .
\end{gathered}
$$

Consider the set $C \cap\{z:|z| \leq \gamma / 2\}$. Smooth out the corners located on $\{z:|z|=\gamma / 2\}$ and call the resulting set $\widetilde{H}$. Define $R_{\delta}=f_{\delta}(\widetilde{H})$.

Let $g_{\delta}$ be the one-to-one conformal mapping (with conformal inverse $g_{\delta}^{-1}$ ) taking $R_{\delta} \backslash\{0\}$ onto $D \backslash\{0\}$ where $D=\{\zeta:|\zeta-1| \leq 1\}$ with $g_{\delta}(0)=0$. Such a map exists because $\partial R_{\delta} \backslash\{0\}$ is $C^{\infty}$. Moreover, $g_{\delta}$ and $g_{\delta}^{-1}$ are continuous on $R_{\delta}$ and $D$, respectively. The proof of the following theoiem about $g_{\delta}^{\prime}$ is very technical, so we leave it for the next section.

Theorem 6.2. If $\delta=\beta$ or if $\delta>2 \beta-1$ then $g_{\delta}^{\prime}$ on $R_{\delta} \backslash\{0\}$ has a continuous extension to $R_{\delta}$ and $g_{\delta}^{\prime} \neq 0$ on $R_{\delta}$. In particular, $\left(g_{\delta}^{-1}\right)^{\prime}$ is boundea on $D$ and $g_{\delta}^{\prime}$ is bounded on $R_{\delta}$.

Remark. When $\delta>\beta$ and $\delta \leq 2 \beta-1$, using results of Warschawski [9] it is possible to show Theorem 6.2 is not true.

In view of Theorem 6.2 , if $\delta=\beta$ or $\delta>2 \beta-1$,

$$
\begin{aligned}
& \left|g_{\delta}^{-1}(\zeta)\right| \leq c|\zeta|, \quad \zeta \in D, \\
& \left|g_{\delta}(w)\right| \leq c|w|, \quad w \in R_{\delta} .
\end{aligned}
$$

Next, map $D \backslash\{(2,0)\}$ onto $S=\{\xi: \operatorname{Im} \xi \geq 0\}$ by the confor:nal mapping

$$
p(\zeta)=\frac{\zeta e^{i \pi / 2}}{2-\zeta}, \quad \zeta \in D \backslash\{(2,0)\}
$$

Also,

$$
p^{-1}(\xi)=\frac{2 \xi}{\xi+e^{i \pi / 2}}, \quad \xi \in S,
$$

is also conformal and we can choose $\varepsilon>0$ such that

$$
f_{\delta}^{-1} \circ g_{\delta}^{-1} \circ p^{-1}\left(\partial S \cap \overline{B_{\varepsilon}(0)}\right) \subseteq \partial C ;
$$

then

$$
\left(p^{-1}\right)^{\prime} \text { is bounded on } \overline{B_{\varepsilon}(0)} \cap S
$$

$$
\frac{1}{c}|\xi| \leq\left|p^{-1}(\xi)\right| \leq c|\xi| \quad \text { for } \xi \in \overline{B_{\varepsilon}(0)} \cap S ;
$$




$$
p^{\prime} \text { is bounded on } p^{-1}\left(\overline{B_{\varepsilon}(0)} \cap S\right) \text {. }
$$

At last we can define $H$ and $F$. Set

$$
H=f_{\delta}^{-1} \circ g_{\delta}^{-1} \circ p^{-1}\left(\overline{B_{\varepsilon}(0)} \cap S\right), \quad F(z)=p \circ g_{\delta} \circ f_{\delta}(z), \quad z \in H .
$$

Then $F$ is conformal from $H \backslash\{0\}$ into $\overline{B_{\varepsilon}(0)} \cap S \backslash\{0\}$ with conformal inverse $\mathscr{F}=f_{\delta}^{-1} \circ g_{\delta}^{-1} \circ p^{-1}$ and parts (i)-(iii) of Theorem 2.1 clearly hold.

By (6.9) and (6.11)

$$
\left|g_{\delta}^{-1} \circ p^{-1}(\xi)\right| \geq c|\xi|, \quad \xi \in \overline{B_{\varepsilon}(0)} \cap S .
$$

Hence for $\xi \in \overline{B_{\varepsilon}(0)} \cap S$, by (6.7), Theorem 6.2 and (6.10)

$$
\begin{aligned}
\left|\mathscr{F}^{\prime}(\xi)\right| & =\left|\left(f_{\delta}^{-1}\right)^{\prime} \circ g_{\delta}^{-1} \circ p^{-1}(\xi)\right| \cdot\left|\left(g_{\delta}^{-1}\right)^{\prime} \circ p^{-1}(\xi)\right| \cdot\left|\left(p^{-1}\right)^{\prime}(\xi)\right| \\
& \leq\left[c\left|g_{\delta}^{-1} \circ p^{-1}(\xi)\right|^{-1}\left(-\ln \left|g_{\delta}^{-1} \circ p^{-1}(\xi)\right|\right)^{-\frac{\beta}{\beta-1}}\right] \cdot[c] \cdot[c] .
\end{aligned}
$$

Since $x \rightarrow \frac{1}{x}(-\ln x)^{-\beta /(\beta-1)}$ is decreasing for $x$ small and positive, by (6.13) we get

$$
\left|\mathscr{F}^{\prime}(\xi)\right| \leq c|\xi|^{-1}(-\ln |\xi|)^{-\frac{\beta}{\beta-1}} \quad \text { for } \xi \in \overline{B_{\varepsilon}(0)} \cap S .
$$

This gives (iv) in Theorem 2.1.

If $z \in H \backslash\{0\}$, then by (6.12), Theorem 6.2 and (6.6)

$$
\begin{aligned}
\left|F^{\prime}(z)\right| & =\left|p^{\prime} \circ g_{\delta} \circ f_{\delta}(z)\right| \cdot\left|g_{\delta}^{\prime} \circ f_{\delta}(z)\right| \cdot\left|f_{\delta}^{\prime}(z)\right| \\
& \leq c \cdot c \cdot c\left|f_{\delta}(z)\right| \cdot|z|^{-\beta}
\end{aligned}
$$

and by (6.4) the latter is bounded. This gives part $(\mathrm{v})$.

All that remains is part (vi). By (6.11) and (6.8)-(6.9)

$$
\frac{1}{c}\left|f_{\delta}(z)\right| \leq|F(z)| \leq c\left|f_{\delta}(z)\right|, \quad z \in H .
$$

Thus for $z \in H \backslash\{0\}$

$$
\begin{aligned}
\left|\frac{F^{\prime}(z)}{F(z)}\right|(-\ln |F(z)|)^{-\frac{\beta}{\beta-1}} & \leq \frac{c\left|f_{\delta}(z)\right| \cdot|z|^{-\beta}}{c\left|f_{\delta}(z)\right|}\left(-\ln c\left|f_{\delta}(z)\right|\right)^{-\frac{\beta}{\beta-1}} \\
& \leq c|z|^{-\beta}\left(-\ln \left|f_{\delta}(z)\right|\right)^{-\frac{\beta}{\beta-1}} \\
& \leq c|z|^{-\beta}\left[c|z|^{-(\beta-1)}\right]^{-\frac{\beta}{\beta-1}} \quad(\text { by }(6.4)) \\
& =c .
\end{aligned}
$$

Here too we are using that the neighborhood $N$ is chosen small enough so that $\theta=\arg z$ is sufficiently small that $\cos ((\beta-1) \theta) \geq c^{\prime}>0$ in (6.4).

\section{Proof of TheOREM 6.2}

We need the following result of Warschawski [10].

Theorem 7.1 (Warschawski). Suppose $\mathscr{C}$ is a closed rectifiable Jordan curve with a continuously turning tangent. Furthermore, assume that the tangent angle $\tau(s)$ as a function of arclength $s$ is Dini continuous. If $G$ maps $D^{0}$ conformally and injectively onto the interior of $\mathscr{C}$ then $G^{\prime}$ has a continuous extension to $D$ and $G^{\prime} \neq 0$ on $D$. 
Here $\tau(s)$ Dini continuous means for some nondecreasing continuous function $\kappa(t), t \in[0, \sigma]$, with

$$
\int_{0}^{\sigma} \frac{\kappa(u)}{u} d u<\infty
$$

we have

$$
\left|\tau\left(s_{2}\right)-\tau\left(s_{1}\right)\right| \leq \kappa\left(\left|s_{2}-s_{1}\right|\right), \quad\left|s_{2}-s_{1}\right| \leq \sigma .
$$

Theorem 6.2 immediately follows from Theorem 7.1 and the next theorem.

Theorem 7.2. If $\delta=\beta$ or $\delta>2 \beta-1$ then $\partial R_{\delta}$ has a continuously turning tangent. The tangent angle $\tau(s)$ is Dini continuous as a function of arclength $s$. Proof. Since $\partial R_{\delta} \backslash\{0\}$ is $C^{\infty}$, we need only consider $\partial R_{\delta}$ in a neighborhood of 0 . Near $0, \partial R_{\delta}$ consists of two arcs, call them $\Gamma_{1}(\delta)$ and $\Gamma_{2}(\delta)$, separated by 0 . We parametrize as follows. Let

$$
\begin{cases}r_{1}(t)=t\left(1+t^{2(\delta-1)}\right)^{1 / 2}, & \theta_{1}(t)=-\tan ^{-1} t^{\delta-1} \\ r_{2}(t)=t\left(1+t^{2(\beta-1)}\right)^{1 / 2}, & \theta_{2}(t)=\tan ^{-1} t^{\beta-1} .\end{cases}
$$

These give parametric representations (in polar coordinates) of $\partial C_{1}, \partial C_{2}$, respectively, for $t \geq 0$. Then for some $\delta_{1}>0$, in polar coordinates $\rho e^{i \varphi}$ for $k=1,2$,

$$
\Gamma_{k}(\delta): \rho=\rho_{k}(t), \quad \varphi=\varphi_{k}(t), \quad t \in\left(0, \delta_{1}\right),
$$

where (cf. (6.4))

$$
\left\{\begin{array}{l}
\rho_{k}(t)=\left|f_{\delta}\left(r_{k}(t) e^{i \theta_{k}(t)}\right)\right| \\
\varphi_{k}(t)=\varphi_{\delta}\left(r_{k}(t) e^{i \theta_{k}(t)}\right)
\end{array}\right.
$$

Then

$$
w(t)= \begin{cases}w_{1}(t)=\rho_{1}(-t) e^{i \varphi_{1}(-t)}, & t \in\left(-\delta_{1}, 0\right), \\ w_{2}(t)=\rho_{2}(t) e^{i \varphi_{2}(t)}, & t \in\left(0, \delta_{1}\right), \\ 0, & t=0,\end{cases}
$$

is a continuous parametric representation of $\partial R_{\delta}$ near 0 .

We need the following expansions.

Lemma 7.3. For $0 \leq t \leq \delta_{1}$,

$$
\begin{aligned}
& \varphi_{1}(t)=-\frac{\pi}{2}+t^{\delta-\beta} \sum_{m=0}^{\infty} a_{m}(\delta) t^{2 m(\delta-1)}, \quad\left\{\begin{array}{l}
a_{0}(\delta)=0, a_{1}(\delta)>0 \text { when } \delta=\beta, \\
a_{0}(\delta)<0 \text { when } \delta>\beta,
\end{array}\right. \\
& \varphi_{2}(t)=\frac{\pi}{2}+\sum_{m=1}^{\infty} b_{m}(\delta) t^{2 m(\beta-1)}, \quad b_{1}(\delta)<0 ; \\
& \frac{\varphi_{1}^{\prime}(t) \rho_{1}(t)}{\rho_{1}^{\prime}(t)}=t^{\delta-1} \sum_{m=0}^{\infty} c_{m}(\delta) t^{2 m(\delta-1)}, \quad\left\{\begin{array}{l}
c_{0}(\delta)=0 \text { if } \delta=\beta, \\
c_{0}(\delta) \neq 0 \text { if } \delta>\beta,
\end{array}\right. \\
& \frac{\varphi_{2}^{\prime}(t) \rho_{2}(t)}{\rho_{2}^{\prime}(t)}=t^{3(\beta-1)} \sum_{m=0}^{\infty} d_{m}(\delta) t^{2 m(\beta-1)}, \quad d_{0}(\delta) \neq 0 .
\end{aligned}
$$


Proof. Since $\tan ^{-1} z$ is analytic on $|z|<1$ and $\sin z$ is entire, $\sin \left(\gamma \tan ^{-1} z\right)$ has a power series on $|z|<1$ in odd powers of $z$. Thus for $\gamma>0$ and $a>0$,

$$
\left(1+u^{2}\right)^{-a} \sin \left(\gamma \tan ^{-1} u\right)=u \sum_{m=0}^{\infty} a_{m}(a, \gamma) u^{2 m}, \quad-1<u<1
$$

where

$$
a_{0}(a, \gamma)=\gamma, \quad a_{1}(a, \gamma)<0 .
$$

Hence by (7.1) and (6.5)

$$
\begin{aligned}
\varphi_{\beta}\left(r_{1}(t) e^{i \theta_{1}(t)}\right) & =-\frac{\pi}{2(\beta-1)} t^{-(\beta-1)} t^{\delta-1} \sum_{m=0}^{\infty} a_{m}\left(\frac{\beta-1}{2}, \beta-1\right) t^{2 m(\delta-1)} \\
& =t^{\delta-\beta}\left[-\frac{\pi}{2}-\sum_{m=1}^{\infty} \tilde{a}_{m}\left(\frac{\beta-1}{2}, \beta-1\right) t^{2 m(\delta-1)}\right], \quad \tilde{a}_{1}<0 .
\end{aligned}
$$

When $\delta=\beta$, by (7.2) this gives

$$
\varphi_{1}(t)=-\frac{\pi}{2}+\sum_{m=1}^{\infty} a_{m}(\beta) t^{2 m(\beta-1)}, \quad a_{1}(\beta)>0 ;
$$

when $\delta>\beta$ we get

$$
\begin{aligned}
\varphi_{1}(t) & =2 t^{\delta-\beta}\left[-\frac{\pi}{2}-\sum_{m=1}^{\infty} \tilde{a}_{m}\left(\frac{\beta-1}{2}, \beta-1\right) t^{2 m(\delta-1)}\right]-\frac{\pi}{2} \\
& =-\frac{\pi}{2}+t^{\delta-\beta} \sum_{m=0}^{\infty} a_{m}(\delta) t^{2 m(\delta-1)}, \quad a_{0}(\delta)<0,
\end{aligned}
$$

as desired.

By (7.1), (7.4) and (6.5)

$$
\varphi_{\beta}\left(r_{2}(t) e^{i \theta_{2}(t)}\right)=\frac{\pi}{2(\beta-1)} \sum_{m=0}^{\infty} a_{m}\left(\frac{\beta-1}{2}, \beta-1\right) t^{2 m(\beta-1)} .
$$

Together with (7.2) this gives for $\delta=\beta$,

$$
\varphi_{2}(t)=\frac{\pi}{2}+\sum_{m=1}^{\infty} b_{m}(\delta) t^{2 m(\beta-1)}, \quad b_{1}(\delta)<0 ;
$$

for $\delta>\beta$,

$$
\begin{aligned}
\varphi_{2}(t) & =2\left[\frac{\pi}{2(\beta-1)} \sum_{m=0}^{\infty} a_{m}\left(\frac{\beta-1}{2}, \beta-1\right) t^{2 m(\beta-1)}\right]-\frac{\pi}{2} \\
& =\frac{\pi}{2}+\sum_{m=1}^{\infty} b_{m}(\delta) t^{2 m(\beta-1)}, \quad b_{1}(\delta)<0,
\end{aligned}
$$

as desired. 
By the chain rule

$$
\begin{aligned}
\varphi_{1}^{\prime}(t)= & (\delta-\beta) t^{\delta-\beta-1} \sum_{m=0}^{\infty} a_{m}(\delta) t^{2 m(\delta-1)} \\
& +t^{\delta-\beta}(\delta-1) t^{\delta-2} \sum_{m=0}^{\infty} a_{m}(\delta) 2 m t^{(2 m-1)(\delta-1)} \\
= & t^{\delta-\beta-1} \sum_{m=0}^{\infty} e_{m}(\delta) t^{2 m(\delta-1)}, \quad \begin{cases}e_{0}(\delta)=0 & \text { if } \delta=\beta, \\
e_{0}(\delta) \neq 0 & \text { if } \delta>\beta,\end{cases}
\end{aligned}
$$

and

$$
\begin{aligned}
\varphi_{2}^{\prime}(t) & =t^{\beta-2} \sum_{m=1}^{\infty}(\beta-1) 2 m b_{m}(\delta) t^{(2 m-1)(\beta-1)} \\
& =t^{-1} \sum_{m=1}^{\infty} f_{m}(\delta) t^{2 m(\beta-1)}, \quad f_{1}(\delta) \neq 0 .
\end{aligned}
$$

An argument similar to that giving (7.4) shows for $\gamma$ and $a>0$,

$$
\left(1+u^{2}\right)^{-a} \cos \left[\gamma \tan ^{-1} u\right]=\sum_{m=0}^{\infty} b_{m}(a, \gamma) u^{2 m}, \quad-1<u<1,
$$

where

$$
b_{0}(a, \gamma)=1
$$

Thus by (6.4) and (7.1)

$$
\begin{aligned}
\left|f_{\beta}\left(r_{1}(t) e^{i \theta_{1}(t)}\right)\right| & =\exp \left\{-\frac{\pi}{2(\beta-1)} t^{-(\beta-1)} \sum_{m=0}^{\infty} b_{m}\left(\frac{\beta-1}{2}, \beta-1\right) t^{2 m(\delta-1)}\right\} \\
& =\exp \left\{t^{-(\beta-1)} \sum_{m=0}^{\infty} g_{m} t^{2 m(\delta-1)}\right\}, \quad g_{0}<0
\end{aligned}
$$

moreover, by the chain rule

$$
\begin{aligned}
\frac{\frac{d}{d t}\left|f_{\beta}\left(r_{1}(t) e^{i \theta_{1}(t)}\right)\right|}{\left|f_{\beta}\left(r_{1}(t) e^{i \theta_{1}(t)}\right)\right|}= & -(\beta-1) t^{-\beta} \sum_{m=0}^{\infty} g_{m} t^{2 m(\delta-1)} \\
& +t^{-(\beta-1)}(\delta-1) t^{\delta-2} \sum_{m=0}^{\infty} 2 m g_{m} t^{(2 m-1)(\delta-1)} \\
= & t^{-\beta} \sum_{m=0}^{\infty} h_{m}(\delta) t^{2 m(\delta-1)}, \quad h_{0}(\delta) \neq 0 .
\end{aligned}
$$

Together with (7.2) and (6.4) this yields

$$
\frac{\rho_{1}(t)}{\rho_{1}^{\prime}(t)}=t^{\beta} \sum_{m=0}^{\infty} j_{m}(\delta) t^{2 m(\delta-1)}, \quad j_{0}(\delta) \neq 0 .
$$

Similarly,

$$
\frac{\rho_{2}(t)}{\rho_{2}^{\prime}(t)}=t^{\beta} \sum_{m=0}^{\infty} k_{m}(\delta) t^{2 m(\beta-1)}, \quad k_{0}(\delta) \neq 0
$$


The formulas (7.6), (7.7), (7.10) and (7.11) yield the desired representation of $\varphi_{i}^{\prime} \rho_{i} / \rho_{i}^{\prime}, i=1,2$.

We continue with the proof of Theorem 7.2. Write

$$
x_{k}(t)=\operatorname{Re} \rho_{k}(t) e^{i \varphi_{k}(t)}, \quad y_{k}(t)=\operatorname{Im} \rho_{k}(t) e^{i \varphi_{k}(t)} .
$$

Then

$$
\frac{x_{k}^{\prime}(t)}{y_{k}^{\prime}(t)}=\frac{\cos \varphi_{k}(t)-\frac{\rho_{k}(t)}{\rho_{k}^{\prime}(t)} \varphi_{k}^{\prime}(t) \sin \varphi_{k}(t)}{\sin \varphi_{k}(t)+\frac{\rho_{k}(t)}{\rho_{k}^{\prime}(t)} \varphi_{k}^{\prime}(t) \cos \varphi_{k}(t)} .
$$

By Lemma 7.3, as $t \rightarrow 0^{+}$,

$$
\begin{aligned}
& \cos \varphi_{1}(t)=\sin \left[t^{\delta-\beta} \sum_{m=0}^{\infty} a_{m}(\delta) t^{2 m(\delta-1)}\right] \\
& = \begin{cases}a_{1}(\delta) t^{2(\beta-1)}+o\left(t^{2(\beta-1)}\right) & \text { if } \delta=\beta, \\
a_{0}(\delta) t^{\delta-\beta}+o\left(t^{\delta-\beta}\right) & \text { if } \delta>\beta,\end{cases} \\
& \sin \varphi_{1}(t)=-1+o(1), \\
& \frac{\rho_{1}(t)}{\rho_{1}^{\prime}(t)} \varphi_{1}^{\prime}(t)= \begin{cases}O\left(t^{3(\beta-1)}\right) & \text { if } \delta=\beta, \\
c_{0}(\delta) t^{\delta-1}+o\left(t^{\delta-1}\right) & \text { if } \delta>\beta,\end{cases} \\
& = \begin{cases}o\left(t^{2(\beta-1)}\right) & \text { if } \delta=\beta, \\
o\left(t^{\delta-\beta}\right) & \text { if } \delta>\beta,\end{cases} \\
& \cos \varphi_{2}(t)=-\sin \sum_{m=1}^{\infty} b_{m}(\delta) t^{2 m(\beta-1)}=-b_{1}(\delta) t^{2(\beta-1)}+o\left(t^{2(\beta-1)}\right), \\
& \sin \varphi_{2}(t)=1+o(1), \\
& \frac{\rho_{2}(t)}{\rho_{2}^{\prime}(t)} \varphi_{2}^{\prime}(t)=d_{0}(\delta) t^{3(\beta-1)}+o\left(t^{3(\beta-1)}\right) .
\end{aligned}
$$

Then as $t \rightarrow 0^{+}$,

$$
\begin{aligned}
\frac{x_{1}^{\prime}(t)}{y_{1}^{\prime}(t)} & = \begin{cases}\frac{a_{1}(\delta) t^{2(\beta-1)}+o\left(t^{2(\beta-1)}\right)-o\left(t^{2(\beta-1)}\right)(-1+o(1))}{-1+o(1)+o(1)}, & \delta=\beta, \\
\frac{a_{0}(\delta) t^{\delta-\beta}+o\left(t^{\delta-\beta}\right)-o\left(t^{\delta-\beta}\right)(-1+o(1))}{-1+o(1)+o(1)}, & \delta>\beta,\end{cases} \\
& \sim \begin{cases}-a_{1}(\delta) t^{2(\beta-1)} \text { if } \delta=\beta, \\
-a_{0}(\delta) t^{\delta-\beta} & \text { if } \delta>\beta,\end{cases} \\
& \rightarrow \begin{cases}0^{-}, & \delta=\beta, \\
0^{+}, & \delta>\beta,\end{cases}
\end{aligned}
$$

and

$$
\begin{aligned}
\frac{x_{2}^{\prime}(t)}{y_{2}^{\prime}(t)} & =\frac{-b_{1}(\delta) t^{2(\beta-1)}+o\left(t^{2(\beta-1)}\right)-d_{0}(\delta) t^{3(\beta-1)}+o\left(t^{3(\beta-1)}\right)}{1+o(1)+o(1)} \\
& \sim-b_{1}(\delta) t^{2(\beta-1)} \\
& \rightarrow 0^{+} .
\end{aligned}
$$

These show that the tangents to $w(t)$ in (7.3) for $t \neq 0$ turn continuously to a vertical line as $t \rightarrow 0$. Thus $\partial R_{\delta}$ has a continuously turning tangent. 
Let $s(t), t \in\left(-\delta_{1}, \delta_{1}\right)$, denote arclength; to prove Dini continuity of $\tau(\cdot)$ as a function of $s$ it suffices to find a continuous nondecreasing function $\kappa$ such that for $\Theta(t)=\tau(s(t))$,

$$
\int_{0^{+}} \frac{\kappa(u)}{u} d u<\infty
$$

and

$$
\left|\boldsymbol{\Theta}\left(t_{1}\right)-\boldsymbol{\Theta}\left(t_{2}\right)\right| \leq \kappa\left(\left|s\left(t_{1}\right)-s\left(t_{2}\right)\right|\right), \quad\left\{\begin{array}{c}
t_{1} \& t_{2} \in\left[0, \delta_{1}\right) \\
\text { OR } \\
t_{1} \& t_{2} \in\left(-\delta_{1}, 0\right]
\end{array}\right.
$$

By (7.13)-(7.14) and (7.3)

$$
\left|\boldsymbol{\Theta}\left(t_{1}\right)-\boldsymbol{\Theta}\left(t_{2}\right)\right|= \begin{cases}\left|\tan ^{-1} \frac{y_{1}^{\prime}}{x_{1}^{\prime}}\left(-t_{1}\right)-\tan ^{-1} \frac{y_{1}^{\prime}}{x_{1}^{\prime}}\left(-t_{2}\right)\right|, & t_{1} \& t_{2} \in\left(-\delta_{1}, 0\right], \\ \left|\tan ^{-1} \frac{y_{2}^{\prime}}{x_{2}^{\prime}}\left(t_{1}\right)-\tan ^{-1} \frac{y_{2}^{\prime}}{x_{2}^{\prime}}\left(t_{2}\right)\right|, & t_{1} \& t_{2} \in\left[0, \delta_{1}\right) .\end{cases}
$$

Writing $\tan ^{-1} a-\tan ^{-1} b=\int_{a}^{b}\left(1+x^{2}\right)^{-1} d x$ for $a, b$ both positive or both negative, we have

$$
\left|\tan ^{-1} \frac{1}{u}-\tan ^{-1} \frac{1}{v}\right| \leq|u-v| \text { for } u \& v>0 \quad \text { OR } \quad u \& v<0 .
$$

Then to prove (7.16), it suffices to show

$$
\left|\frac{x_{i}^{\prime}}{y_{i}^{\prime}}\left(t_{1}\right)-\frac{x_{i}^{\prime}}{y_{i}^{\prime}}\left(t_{2}\right)\right| \leq \kappa\left(\int_{t_{1}}^{t_{2}} \sqrt{x_{i}^{\prime}(u)^{2}+y_{i}^{\prime}(u)^{2}} d u\right), \quad 0 \leq t_{1}<t_{2}<\delta_{1} .
$$

For typographical clarity in the next few lines we drop the $i$ subscript. By (7.12) for

$$
h(u)=\sin \varphi(u)+\varphi^{\prime}(u) \frac{\rho(u)}{\rho^{\prime}(u)} \cos \varphi(u),
$$

we have

$$
\begin{aligned}
\mid \frac{x^{\prime}}{y^{\prime}}\left(t_{1}\right)- & \frac{x^{\prime}}{y^{\prime}}\left(t_{2}\right) \mid \\
\leq & \left\{\left|\left[1+\varphi^{\prime}\left(t_{1}\right) \varphi^{\prime}\left(t_{2}\right) \frac{\rho\left(t_{1}\right) \rho\left(t_{2}\right)}{\rho^{\prime}\left(t_{1}\right) \rho^{\prime}\left(t_{2}\right)}\right] \sin \left(\varphi\left(t_{2}\right)-\varphi\left(t_{1}\right)\right)\right|\right. \\
& \left.+\left|\left[\varphi^{\prime}\left(t_{2}\right) \frac{\rho\left(t_{2}\right)}{\rho^{\prime}\left(t_{2}\right)}-\varphi^{\prime}\left(t_{1}\right) \frac{\rho\left(t_{1}\right)}{\rho^{\prime}\left(t_{1}\right)}\right] \cos \left(\varphi\left(t_{2}\right)-\varphi\left(t_{1}\right)\right)\right|\right\} \cdot\left|h\left(t_{1}\right) h\left(t_{2}\right)\right|^{-1} .
\end{aligned}
$$

By the formulas after (7.12), $|h|$ is bounded below away from 0 for $\delta_{1}$ small and $1+\varphi^{\prime}\left(t_{1}\right) \varphi^{\prime}\left(t_{2}\right) \rho\left(t_{1}\right) \rho\left(t_{2}\right) / \rho^{\prime}\left(t_{1}\right) \rho^{\prime}\left(t_{2}\right)$ is bounded. Therefore

$$
\left|\frac{x^{\prime}}{y^{\prime}}\left(t_{1}\right)-\frac{x^{\prime}}{y^{\prime}}\left(t_{2}\right)\right| \leq C\left[\left|\varphi\left(t_{2}\right)-\varphi\left(t_{1}\right)\right|+\left|\varphi^{\prime}\left(t_{2}\right) \frac{\rho\left(t_{2}\right)}{\rho^{\prime}\left(t_{2}\right)}-\varphi^{\prime}\left(t_{1}\right) \frac{\rho\left(t_{1}\right)}{\rho^{\prime}\left(t_{1}\right)}\right|\right] .
$$


Now we examine each term separately. By Lemma 7.3

$$
\begin{aligned}
\left|\varphi_{1}\left(t_{2}\right)-\varphi_{1}\left(t_{1}\right)\right| & =\left|\sum_{m=0}^{\infty} a_{m}(\delta)\left[t_{2}^{2 m(\delta-1)+\delta-\beta}-t_{1}^{2 m(\delta-1)+\delta-\beta}\right]\right|, \\
\left|\varphi_{2}\left(t_{2}\right)-\varphi_{2}\left(t_{1}\right)\right| & =\left|\sum_{m=1}^{\infty} b_{m}(\delta)\left[t_{2}^{2 m(\beta-1)}-t_{1}^{2 m(\beta-1)}\right]\right|, \\
\left|\varphi_{1}^{\prime} \frac{\rho_{1}}{\rho_{1}^{\prime}}\left(t_{2}\right)-\varphi_{1}^{\prime} \frac{\rho_{1}}{\rho_{1}^{\prime}}\left(t_{1}\right)\right| & =\left|\sum_{m=0}^{\infty} c_{m}(\delta)\left[t_{2}^{(2 m+1)(\delta-1)}-t_{1}^{(2 m+1)(\delta-1)}\right]\right|, \\
\left|\varphi_{2}^{\prime} \frac{\rho_{2}}{\rho_{2}^{\prime}}\left(t_{2}\right)-\varphi_{2}^{\prime} \frac{\rho_{2}}{\rho_{2}^{\prime}}\left(t_{2}\right)\right| & =\left|\sum_{m=0}^{\infty} d_{m}(\delta)\left[t_{2}^{(2 m+3)(\beta-1)}-t_{1}^{(2 m+3)(\beta-1)}\right]\right| .
\end{aligned}
$$

Choose $r \in(0,1]$ so small that $\delta-(2 \beta-1)-r(\beta-1)>0$ if $\delta>\beta$; otherwise let $r=1$. Here is where we use the hypothesis $2 \beta-1<\delta$ whenever $\delta>\beta$. This particular choice of $r$ does not come into play until the end of this section. Define

$$
\kappa(u)=(-\ln u)^{-(1+r)}, \quad 0<u<\frac{1}{2} .
$$

Then $\kappa(u)$ is increasing and satisfies (7.15). We need the following lemma to examine the right side of (7.17).

Lemma 7.4. Assume $a, p, \eta, b>0$. Then for some $b_{0}, c>0$ independent of $\eta$,

$$
\sup _{x>1}\left(1-x^{-p}\right)^{a}\left(\eta-\ln \left[1-e^{-b(x-1)}\right]\right) \leq \eta \vee c b
$$

whenever $b \geq b_{0}$.

Proof. Call the function to be maximized $k(x)$. If $2>\frac{p+1}{b}$, the function $x^{p+1} e^{-b(x-1)}$ is decreasing for $x \geq 2$. Hence for such $b$,

$$
\sup _{x \geq 2} x^{p+1} e^{-b(x-1)}=2^{p+1} e^{-b}
$$

Consider the function

$$
k_{1}(x)=\frac{1-x^{-p}}{1-e^{-b(x-1)}}, \quad 1<x \leq 2 .
$$

Since $\lim _{x \rightarrow 1^{+}} k_{1}(x)=\frac{p}{b}$ and $k_{1}(2) \leq\left(1-e^{-b}\right)^{-1}$,

$$
\sup _{1<x \leq 2} k_{1}(x)=\frac{p}{b} \vee \frac{1}{1-e^{-b}} \vee \sup \left\{k_{1}\left(x_{0}\right): 1<x_{0}<2, k_{1}^{\prime}\left(x_{0}\right)=0\right\} \text {. }
$$

But if $k_{1}^{\prime}\left(x_{0}\right)=0$ then

$$
e^{-b\left(x_{0}-1\right)}=\frac{p x_{0}^{-p-1}}{p x_{0}^{-p-1}+b\left(1-x_{0}^{-p}\right)}
$$

and so

So we get

$$
k_{1}\left(x_{0}\right)=\frac{p x_{0}^{-p-1}+b\left(1-x_{0}^{-p}\right)}{b}<\frac{p+b}{b} \text {. }
$$

$$
\sup _{1<x \leq 2} k_{1}(x) \leq \frac{p+b}{b} \vee \frac{1}{1-e^{-b}}
$$


Using (7.20)-(7.22), for some $b_{0}$,

$$
\sup _{x>1} \frac{\left(1-x^{-p}\right)^{a+1}}{1-e^{-b(x-1)}} x^{p+1} e^{-b(x-1)}=c<\infty \quad \text { if } b \geq b_{0} .
$$

Since

$$
\lim _{x \rightarrow 1^{+}} k(x)=0, \quad \lim _{x \rightarrow \infty} k(x)=\eta
$$

we have

$$
\sup _{x>1} k(x)=\eta \vee \sup \left\{k\left(x_{0}\right): x_{0}>1 \text { and } k^{\prime}\left(x_{0}\right)=0\right\} \text {. }
$$

If $x_{0}>1$ and $k^{\prime}\left(x_{0}\right)=0$ then

$$
\eta-\ln \left[1-e^{-b\left(x_{0}-1\right)}\right]=\frac{b}{a p} x_{0}^{p+1} e^{-b\left(x_{0}-1\right)} \frac{1-x_{0}^{-p}}{1-e^{-b\left(x_{0}-1\right)}}
$$

and so

$$
\begin{aligned}
k\left(x_{0}\right) & =\frac{\left(1-x_{0}^{-p}\right)^{a+1}}{1-e^{-b\left(x_{0}-1\right)}} \frac{b}{a p} x_{0}^{p+1} e^{-b\left(x_{0}-1\right)} \\
& \leq \frac{c}{a p} b \quad \text { provided } b \geq b_{0}
\end{aligned}
$$

by (7.23). Thus

$$
\sup _{x>1} k(x) \leq \eta \vee c b, \quad b \geq b_{0} \text { as desired. }
$$

By (7.10)-(7.11), (7.2) and (6.4), for some $\gamma_{i}>0$, if $t$ is sufficiently small,

and since

$$
\left|\rho_{i}^{\prime}(t)\right| \geq c t^{-\beta} \exp \left(-\gamma_{i} t^{-(\beta-1)}\right),
$$

$$
\begin{aligned}
\sqrt{x_{i}^{\prime}(u)^{2}+y_{i}^{\prime}(u)^{2}} & =\left|\rho_{i}^{\prime}(u)\right| \sqrt{1+\left[\varphi_{i}^{\prime} \frac{\rho_{i}}{\rho_{i}^{\prime}}(u)\right]^{2}} \\
& \geq c u^{-\beta} \exp \left(-\gamma_{i} u^{-(\beta-1)}\right)
\end{aligned}
$$

it follows that

$$
\begin{aligned}
\int_{t_{1}}^{t_{2}} \sqrt{x_{i}^{\prime}(u)^{2}+y_{i}^{\prime}(u)^{2}} d u & \geq c \int_{t_{1}}^{t_{2}} u^{-\beta} e^{-\gamma_{i} u^{-(\beta-1)}} d u \\
& =c\left[e^{-\gamma_{i} t_{2}^{-(\beta-1)}}-e^{\left.-\gamma_{i} t_{1}^{-(\beta-1)}\right]}\right. \\
& =c e^{-\gamma_{i} t_{2}^{-(\beta-1)}}\left[1-e^{-\gamma_{i}\left[t_{1}^{-(\beta-1)}-t_{2}^{-(\beta-1)}\right]}\right] .
\end{aligned}
$$

Thus

$$
\begin{aligned}
& \kappa\left(\int_{t_{1}}^{t_{2}} \sqrt{x_{i}^{\prime}(u)^{2}+y_{i}^{\prime}(u)^{2}} d u\right) \\
& \quad \geq\left(-\ln c+\gamma_{i} t_{2}^{-(\beta-1)}-\ln \left[1-e^{-\gamma_{i}\left[t_{1}^{-(\beta-1)}-t_{2}^{-(\beta-1)}\right]}\right]\right)^{-(1+r) .}
\end{aligned}
$$

For $q>0$ (where RHS = Right-hand side), with $0 \leq t_{1}<t_{2}$,

$$
\begin{aligned}
& \frac{t_{2}^{q}-t_{1}^{q}}{\operatorname{RHS}(7.24)}=t_{2}^{q}\left[( 1 - ( \frac { t _ { 1 } } { t _ { 2 } } ) ^ { q } ) ^ { 1 / ( 1 + r ) } \left(-\ln c+\gamma_{i} t_{2}^{-(\beta-1)}\right.\right. \\
& \left.-\ln \left[1-e^{-\gamma_{i} t_{2}^{-(\beta-1)}\left[\left(t_{1} / t_{2}\right)^{-(\beta-1)}-1\right]}\right)\right]^{1+r} \\
& \leq t_{2}^{q}\left\{\left[-\ln c+\gamma_{i} t_{2}^{-(\beta-1)}\right] \vee c \gamma_{i} t_{2}^{-(\beta-1)}\right\}^{1+r} \text { for } t_{2} \text { small, }
\end{aligned}
$$


by Lemma 7.4 (setting $a=1 /(1+r), \eta=-\ln c+\gamma_{i} t_{2}^{-(\beta-1)}, b=\gamma_{i} t_{2}^{-(\beta-1)}$, $p=q /(\beta-1)$, and $\left.x=\left(t_{1} / t_{2}\right)^{-(\beta-1)}>1\right)$. Then for $t_{2}$ small,

$$
\frac{t_{2}^{q}-t_{1}^{q}}{\operatorname{RHS}(7.24)} \leq C t_{2}^{q-(1+r)(\beta-1)}
$$

where $q>0$ and $C$ is independent of $t_{1}$ and $t_{2}$.

Finally, we prove

$$
\frac{\left|\varphi_{1}\left(t_{2}\right)-\varphi_{1}\left(t_{1}\right)\right|}{\operatorname{LHS}(7.24)} \leq C \text { for } 0 \leq t_{1}<t_{2}, t_{2} \text { small }
$$

Similar arguments prove similar bounds for $\left|\varphi_{1}\left(t_{2}\right)-\varphi_{1}\left(t_{1}\right)\right|$ replaced by the left hand parts in the 3 remaining equations in (7.19). Then together with (7.18) we see that (7.17) holds with $\kappa$ replaced by $C \kappa$ and we will be done. So on to $(7.26)$.

Lemma 7.5. If $p \geq 1$ then $\left(1-x^{p}\right) /(1-x) \leq p$ for $0 \leq x \leq 1$.

Corollary 7.6. If $p \geq 1$ then

$$
\left|t_{2}^{p q}-t_{1}^{p q}\right| \leq p\left|t_{2}\right|^{(p-1) q}\left|t_{2}^{q}-t_{1}^{q}\right| .
$$

Choose $q>1$ such that

$$
q-(1+r)(\beta-1)>0 .
$$

Then choose $M \geq 1$ such that

$$
2 m(\beta-1)-q>1 \text { for } m \geq M \text {. }
$$

By Corollary 7.6, (7.19) and (7.25)

$$
\begin{aligned}
\frac{\left|\varphi_{1}\left(t_{2}\right)-\varphi_{1}\left(t_{1}\right)\right|}{\operatorname{LHS}(7.24)} \leq & \frac{\left|\varphi_{1}\left(t_{2}\right)-\varphi_{1}\left(t_{1}\right)\right|}{\operatorname{RHS}(7.24)} \\
\leq & \frac{\left|\sum_{m=0}^{M} a_{m}(\delta)\left[t_{2}^{2 m(\delta-1)+\delta-\beta}-t_{1}^{2 m(\delta-1)+\delta-\beta}\right]\right|}{\operatorname{RHS}(7.24)} \\
& +\frac{\sum_{m=M+1}^{\infty}\left|a_{m}(\delta)\right| t_{2}^{2 m(\delta-1)+\delta-\beta-q}\left[\frac{2 m(\delta-1)+\delta-\beta}{q}\right]\left|t_{2}^{q}-t_{1}^{q}\right|}{\operatorname{RHS}(7.24)} \\
\leq & c \sum_{m=0}^{M}\left|a_{m}(\delta)\right| t_{2}^{2 m(\delta-1)+\delta-\beta-(\beta-1)(1+r)}+c t_{2}^{q-(\beta-1)(1+r)}
\end{aligned}
$$

(by $(7.25)$ )

where we have used (7.28) and (7.25) to bound the second summation. The last term on the RHS is bounded for $t_{2}$ small by (7.27). If $\delta>\beta$ then $2 m(\delta-1)+(\delta-\beta)-(1+r)(\beta-1) \geq \delta-(2 \beta-1)-r(\beta-1)>0$ by choice of $r$. When $\delta=\beta$ then since $a_{0}=0$ we are only concerned with $m \in\{1,2, \ldots, M\}$ in the first summation and then $2 m(\delta-1)+(\delta-\beta)-$ $(1+r)(\beta-1) \geq 0$ by choice of $r$. In any event the first summation is also bounded for $t_{2}$ small. This gives (7.26) and we are done. 


\section{REFERENCES}

1. R. D. DeBlassie, Explicit semimartingale representation of Brownian motion in a wedge, Stochastic Process. Appl. 34 (1990), 67-97.

2. K. Burdzy and D. Marshall, Hitting a boundary point with reflected Brownian motion, (1990), preprint.

3. L. C. G. Rogers, Brownian motion in a wedge with variable skew reflection, Trans. Amer. Math. Soc. 326 (1991), 227-236.

4. __ Brownian motion in a wedge with variable skew reflection. II, Proc. Evanston Conf. (1990), (to appear).

5. L. C. G. Rogers and D. Williams, Diffusions, Markov processes, and martingales, Vol. 2: Itô calculus, Wiley, Chichester, 1987.

6. D. W. Stroock and S. R. S. Varadhan, Diffusion processes with boundary conditions, Comm. Pure. Appl. Math. 24 (1971), 147-225.

7. D. W. Stroock and S. R. S. Varadhan, Multidimensional diffusion processes, Springer-Verlag, New York, 1979.

8. S. R. S. Varadhan and R. J. Williams, Brownian motion in a wedge with oblique reflection, Comm. Pure Appl. Math. 38 (1985), 405-443.

9. E. Warschawski, On conformal mapping of infinite strips, Trans. Amer. Math. Soc. 51 (1942), 280-335.

10. 12 (1961), 614-620.

11. R. J. Williams, Recurrence classification and invariant measure for reflected Brownian motion in a wedge, Ann. Probab. 13 (1985), 758-778.

Department of Mathematics, Texas A\&M University, College Station, Texas 77843

E-mail address: deblass@math.tamu.edu

E-mail address: toby@math.tamu.edu 